\title{
The multiple roles of ubiquitylation in regulating challenged DNA replication.
}

\author{
Sara Villa-Hernández ${ }^{1}$, Avelino Bueno ${ }^{2,3}$ \& Rodrigo Bermejo ${ }^{1^{*}}$. \\ ${ }^{1}$ Centro de Investigaciones Biológicas (CSIC), Madrid, Spain; ${ }^{2}$ Instituto de Biología \\ Molecular y Celular del Cáncer (USAL/CSIC) and ${ }^{3}$ Departamento de Microbiología y \\ Genética, Universidad de Salamanca, Spain \\ *Correspondence to: rodrigo.bermejo@csic.es
}

\begin{abstract}
DNA replication is essential for the propagation of life and the development of complex organisms. However, replication is a risky process as it can lead to mutations and chromosomal alterations. Conditions challenging DNA synthesis by replicative polymerases or DNA helix unwinding, generally termed as replication stress, can halt replication fork progression. Stalled replication forks are unstable and mechanisms exist to protect their integrity, which promote an efficient re-start of DNA synthesis and counteract fork collapse characterized by the accumulation of DNA lesions and mutagenic events. DNA replication is a highly regulated process and several mechanisms control replication timing and integrity both during unperturbed cell cycles and in response to replication stress. Work over the last two decades has revealed that key steps of DNA replication are controlled by conjugation of the small peptide ubiquitin. While ubiquitylation was traditionally linked to protein degradation, the complexity and flexibility of the ubiquitin system in regulating protein function has recently emerged. Here we review the multiple roles exerted by ubiquitin conjugating enzymes and ubiquitin-specific proteases, as well as readers of ubiquitin chains, in the control of eukaryotic DNA replication and replication-coupled DNA damage tolerance and repair.
\end{abstract}




\section{Keywords}

DNA replication, Replication Forks, Replication Stress, DNA damage response, DNA repair, DNA damage tolerance, Ubiquitin, Ubiquitin ligases, DUBs, Cdc48/p97

\section{The ubiquitin system.}

Ubiquitylation is a highly regulated three-enzyme process consisting in the covalent attachment of ubiquitin moieties to a determined substrate. First, an E1 ubiquitin-activating enzyme forms a high-energy thioester bond with the carboxyl group of the terminal glycine residue of ubiquitin (Figure 1a). This activated ubiquitin is then transferred to an E2 ubiquitin-conjugating enzyme by transesterification. An E3 ubiquitin ligase then catalyses the formation of an isopeptide bond between a lysine in the substrate and the activated carboxyl group of ubiquitin (Komander and Rape 2012). Multiple rounds of this process, using lysines on ubiquitin as a substrate, lead to the formation of different types of polyubiquitin chains (Figure 1b). Any of the seven lysines present on ubiquitin (K6, K11, K27, K29, K33, K48 and K63), as well as the amino-terminal methionine (Met1) of the ubiquitin monomer, can serve as isopeptide bond acceptors (Komander and Rape 2012; Kulathu and Komander 2012). Moreover, not only homotypic ubiquitin chains can be formed, but also atypical chains, such as mixed chains (in which different lysines are successively used to link ubiquitin moeties) or branched chains (in which different lysines are ubiquitylated within a given ubiquitin molecule).

Ubiquitin chain variants can occur and determine different outcomes of the modified substrates. While K11 and K48 chains more frequently signal proteins for degradation, monoubiquitylation and K63 chains usually modulate protein-protein interactions. However, these regulatory functions are not strict and proteolytic outcomes of K63 chains and non-proteolytic ones for K48 have been described (Shibata et al. 2012; Maric et al. 2014). K6, K27, K29 and K33-ubiquitin chains have been reported only for a small number of substrates and their function is still 
poorly understood (Kulathu and Komander 2012; Pinder et al. 2013; Yau and Rape 2016). As in the case of other post-translational modifications ubiquitylation can be reversed and this is achieved by the action of specific ubiquitin proteases or deubiquitinases (DUBs). DUBs are cysteine proteases (with the exception of budding yeast Rpn11 that is a zinc metalloprotease), which catalyse the hydrolysis of the isopeptide bonds connecting ubiquitin with its substrate (Nijman et al. 2005b; Sahtoe and Sixma 2015). Ubiquitylation modulates a great variety of cellular processes and is regulated in a more sophisticated way than initially anticipated by factors that promote either substrate ubiquitylation or deubiquitylation. Noteworthy, pairs of coordinated E3 ligases and DUBs have been identified, in which the two enzymes act on the same substrates to fine-tune ubiquitylation levels (Kee et al. 2005; Sowa et al. 2009). Another important layer of regulation comes from ubiquitin chain editing, which requires the concerted action of additional ubiquitin ligases and/or DUBs that change the topology of the ubiquitin chains and thus alter substrate fate (Newton et al. 2008)

\section{Control of unperturbed DNA replication by ubiquitylation.}

DNA replication is initiated at sites of the genome known as origins of replication, to which the Origin Recognition Complex (ORC) binds (Bell and Stillman 1992). ORC is required for the recruitment of the MCM2-7 complex to origin DNA, by the action of Cdc6 and Cdt1 proteins in a process termed origin licensing (Diffley et al. 1994). These sequentially loaded proteins conform the pre-replicative complex (pre-RC), which marks sites that can potentially initiate replication (Figure 2). Upregulation of CDK (Cyclin-dependent kinase) and DDK (Dbf4-dependent kinase) activities after the transition from G1 to S phase leads to the phosphorylation and recruitment of additional factors and the formation of a pre-initiation complex (pre-IC) (Francis et al. 2009; Randell et al. 2010). Among these factors are Cdc45 and the GINS (Go-Ichi-Ni-San) complex, which together with MCM2-7 conform the functional replicative helicase (Ilves et al. 2010), as well as Mcm10, Dpb11 and DNA polymerase $\varepsilon$ (Muramatsu et al. 2010). Once MCM2-7 complex is phosphorylated and activated, the double hexamer divides into two hexamers that 
start unwinding DNA (Quan et al. 2015). Helicase activation generates replication forks to which additional factors are recruited to build replisomes capable of efficient DNA synthesis (Bell and Labib 2016).

Origin licencing and firing are restricted to G1 and S-phase, respectively, in order to ensure that replication occurs once and only once per cell cycle (Siddiqui et al. 2013). This is achieved by coupling origin function to the oscillation in the activity of cyclin-dependent kinases. Origin licensing occurs when CDK levels are low due to degradation of mitotic cyclins and the CDK-activating phosphatase Cdc25, mediated by the anaphase promoting complex (APC) ubiquitin ligase and its adaptor Cdh1 (Donzelli et al. 2002). Additionally, helicase loading to origin DNA is prevented outside of G1 by the degradation of the licensing factors Cdc6 and Cdt1 (Figure 2). Cdc6 is marked for ubiquitin-mediated proteolysis by phosphorylation by CDK upon S-phase onset. In budding yeast, phosphorylated Cdc6 is ubiquitylated by the Skp1-Cullin-F-box SCFSkp2 E3 ligase complex (Sánchez et al. 1999), leading to its degradation and thus prevention of MCM2-7 recruitment. In human cells instead, CDC6 phosphorylation leads to its export to the cytoplasm (Yim et al. 2013), where it cannot exert its MCM chromatin loading function. Nonetheless, it has been recently proposed that human CDC6 undergoes ubiquitylation-mediated degradation in a mechanism reminiscent of the one described in yeast (Walter et al. 2016).

Similarly to Cdc6, the Cdt1 origin-licensing factor is degraded by ubiquitinmediated proteolysis. In human cells, CDT1 can be ubiquitylated by both the E3 Cullin-Ring ubiquitin ligase complex CRL4 ${ }^{\mathrm{Cdt} 2}$ and the SCFSkp2 E3 ligase (Li et al. 2003; Nishitani et al. 2006). CDT1 degradation is induced by its phosphorylation by CDK, which promotes the interaction between CDT1 and Skp2 (Sugimoto et al. 2004). CDT1 degradation is also promoted by ATR checkpoint-dependent phosphorylation of the Cdt2 adaptor in response to DNA damage (Sakaguchi et al. 2012), as part of a mechanism that may contribute to limit origin licensing and firing upon checkpoint activation. In addition, it has been recently reported that the ATM (Ataxia-telangiectasia mutated) checkpoint kinase promotes the degradation of CDT1 during unperturbed replication (Iwahori et al. 2014). 
However, the identity of the signals determining ATM activation and CDT1 degradation in the absence of exogenous perturbations remains unclear. CDT1 is also regulated by deubiquitylation. Cells ablated for the USP37 ubiquitin protease exhibit hyper-ubiquitylated CDT1 and USP37 has been shown to deubiquitylate CDT1 in vitro (Hernández-Pérez et al. 2016). Ablation of USP37 leads to defects in S-phase progression, but whether this reflects alterations in origin licensing or a yet unidentified function for CDT1 is promoting on-going replication remains to be elucidated. In metazoan, CDT1 function is subject to an additional layer of regulation through inhibition by the small nuclear protein Geminin (Wohlschlegel et al. 2000). Geminin is expressed upon transition from G1 to S-phase and is degraded at the end of mitosis through a destruction box targeted by APC (McGarry and Kirschner 1998). Thus, multiple redundant mechanisms control licencing factors function and stability to restrict helicase loading to the G1 phase of the cell cycle.

Ubiquitylation is also important during termination of DNA replication (Figure 3). Both in budding yeast and Xenopus, K48 ubiquitin chains are linked to $\mathrm{Mcm} 7$ during the last steps of replication. Mcm7 ubiquitylation by yeast SCFDia2 or Xenopus CRL2 ${ }^{\text {Lrr1 }}$ ubiquitin ligases leads to the disassembly of the CMG helicase from chromatin by action of the Cdc48/p97 ubiquitin selective segregase (Priego Moreno et al. 2014; Maric et al. 2014; Maculins et al. 2015; Dewar et al. 2017). In this process, ubiquitylation by SCFDia2 does not trigger Mcm7 degradation and appears to be restricted to converging forks through mechanisms that are not yet understood. It had been previously reported that CDC-48/p97 is required for the extraction from chromatin and degradation of CDT-1 in Chaenorabditis embryos and Xenopus extracts (Franz et al. 2011). In this study, accumulation of CDC45/GINS on chromatin was also observed upon ablation of CDC-48 and it was proposed that CDT-1 degradation might be linked to GINS release from chromatin. Cdc48/p97 has also been involved in the extraction of proteins associated to chromatin during replication elongation (Lecona et al. 2016). It was reported that in human cells proteins bound to nascent DNA have higher levels of modification by the SUMO (Small Ubiquitin-like modifier) protein, while ubiquitylation is more abundantly detected in mature chromatin. The replisome-associated USP7 
ubiquitin protease was found to counteract the accumulation of ubiquitylated proteins around nascent strands and thus limit their extraction by Cdc48/p97. Impairment of USP7 function results in fork progression defects, suggesting that limiting the levels of ubiquitylated proteins is important to sustain processive chromatin replication.

\section{Ubiquitin in the modulation of DNA replication in conditions of stress.}

DNA replication can be challenged in conditions that impede replication fork progression, broadly termed as replication stress (Gaillard et al. 2015). Replication stress can result from inhibition of DNA synthesis, as in the case of dNTP pool depletion or polymerase blocking DNA lesions, or be due to blockage of DNA unwinding by replicative helicases, as in the case of inter-strand crosslinks (ICLs) or DNA-protein crosslinks (DPCs). These conditions are generally thought to cause an excessive accumulation of ssDNA, either due to the uncoupling between the replicative DNA polymerases and helicases or owing to nucleolytic resection of replication/repair intermediates (Jossen and Bermejo 2013). Stalled replication forks are unstable and prone to accumulate DNA breaks priming genomic instability (Branzei and Foiani 2010). Several mechanisms have been described that contribute stabilize replication forks and prevent their collapse. A prominent role in preserving genome stability is played by the replication checkpoint, which is mediated by highly conserved kinases Mec1/ATR and Rad53/CHK1 that monitor problems in fork progression. Upon fork stalling, extended ssDNA filaments coated by Replication Protein A (RPA) complex recruit Mec1/ATR through its partner protein Ddc2/ATRIP (Zou and Elledge 2003). At replication forks, Mec1 phosphorylates several targets, which include the Mrc1/CLASPIN protein (Alcasabas et al. 2001; Tanaka and Russell 2001). Mrc1 is a replisome component that acts as a mediator likely facilitating Rad53 in trans auto-phosphorylation leading to its full kinase activation required for the stabilization of replication forks (Alcasabas et al. 2001; Pellicioli and Foiani 2005). Checkpoint kinases interplay with factors dedicated to preserve replisome architecture and functionality, as well as with DNA repair and DNA damage tolerance pathways 
(Branzei and Foiani 2010; Ulrich and Walden 2010). Homologous Recombination (HR) proteins are also required for replication fork stabilization, though their role in this process, likely related to ssDNA protection, is not yet fully understood (Carr and Lambert 2013). DNA lesions blocking the progression of replicative DNA polymerases can be bypassed through DNA damage tolerance (DDT) pathways (Chang and Cimprich 2009), which are driven by error-prone Translesion Synthesis (TLS) and the error-free Template Switching (TS) mechanisms. These are mediated by ubiquitylation of PCNA (Proliferating Cell Nuclear Antigen) and their impairment leads to increased mutagenesis and loss of viability upon treatment with genotoxic agents.

\subsection{Ubiquitylation of single stranded DNA binding proteins at stalled replication forks.}

Replication protein A (RPA) complex is composed of three subunits (Rfa1/RPA70, Rfa2/RP32 and Rfa3/RPA14) and is phosphorylated by checkpoint kinases (Vassin et al. 2009; Ghospurkar et al. 2015). It has been recently described that all RPA subunits are ubiquitylated in response to replication fork stalling induced by agents causing dNTP depletion or DNA damage (Elia et al. 2015). RPA ubiquitylation depends on the RFWD3 E3 ligase and does not induce degradation by the proteasome. RFWD3 depleted cells show defects in stalled fork restart and increased sister chromatid exchange events in conditions inducing fork stalling. Based on these observations, it was proposed that RFWD3-dependent ubiquitylation of RPA promotes homologous recombination-mediated re-start of stalled forks (Figure 4). It was suggested that ubiquitylation of RPA favors HR by promoting displacement of RPA from ssDNA to facilitate RAD51 filament formation. Alternatively, ubiquitylated RPA may provide a signal for attracting RAD51 to stalled replication forks. Previously, RPA subunits ubiquitylation by a different E3 ligase, PRP19, in response to DNA damage had been reported (Maréchal et al. 2013). It was shown that depletion of PRP19 reduced DNA damage-induced ubiquitylation of RPA and a role for this modification in promoting ATR-ATRIP signaling and fork progression was proposed. This 
conclusion was challenged by the finding that the system used to interfere PRP19 expression resulted in a collateral increase of overall levels of exogenously expressed ubiquitin (Elia et al. 2015). Hence, further work will be required to clarify the relative contribution of RFWD3 and PRP19 to RPA ubiquitylation and stalled fork protection.

A role for the ubiquitylation of the Homologous Recombination (HR) mediator RAD51 in response to replication stress conditions has also been proposed. Rad51 is ubiquitylated in vitro by the FBH1 helicase/F-box protein (Chu et al. 2015). FBH1 helicase function had been previously implicated in modulating HRmediated DNA repair as it can displace RAD51 nucleofilaments (Simandlova et al. 2013). Conversely, FBH1 ubiquitin ligase activity has been proposed to limit recombination-driven genomic instability by modulating RAD51 association to stalled forks (Chu et al. 2015). Expression of a RAD51 K58/64R variant, which shows impaired ubiquitylation in vitro, results in increased recombination and promotes viability and fork stability in response to replication stress inducing agents. It was hence proposed that ubiquitylation may counteract RAD51 accumulation at stalled forks, thus limiting unscheduled recombination events (Figure 4). The RecQ-family BLM (Bloom) helicase also plays an important role in the regulation of HR-mediated DNA repair. It has been proposed that BLM exerts both pro- and anti-recombinogenic functions at replication forks, by limiting HR events upon fork stalling or, conversely, promoting Rad51 recruitment to collapsed forks to promote repair (Böhm and Bernstein 2014). BLM function at replication forks is regulated by post-translational modifications. Upon HU treatment, recruitment of BLM to sites of stalled replication requires its polyubiquitylation on three different lysines (K105/K225/K259) by the RNF8 and RNF168 E3 ligases (Tikoo et al. 2013). Expression of non-ubiquitylatable forms of BLM results in increased HR levels. This, and the fact that BLM can disrupt RAD51 nucleofilaments in vitro (Bugreev et al. 2007) lead to the notion that ubiquitylation enhances BLM anti-recombinogenic function at stalled forks (Figure 4). Conversely, BLM sumoylation mediates the pro-recombinogenic role of BLM upon fork collapse, though the underlying mechanism is less characterized (Böhm and Bernstein 2014). Collectively, this evidence hints at ubiquitylation as a mechanism 
to modulate the balance between RPA and Rad51 association to ssDNA and fine tune HR events at stalled replication forks.

\subsection{Crosstalk between DNA damage checkpoint signaling and ubiquitin systems.}

Several lines of evidence point at a regulation of replication stress signaling by the checkpoint response through ubiquitylation. In S. cerevisiae, the Mrc1/CLASPIN adaptor is ubiquitylated by the SCFDia2 E3 ligase in vitro. In line with this observation, Mrc1 is degraded upon induction of DNA damage in S-phase in a manner dependent on Dia2 (Mimura et al. 2009). In Schizosaccharomyces pombe, deletion of the replication fork protection complex factor Swi1 (homolog of Tof1/TIMELESS) induces degradation of Mrc1 dependent on the SCFDia2 homolog SCFPof3 (Roseaulin et al. 2013). This evidence suggests that Mrc1 is degraded through ubiquitylation via Dia2, which is a fork-associated factor. Cells ablated for Dia2 are sensitive to DNA damage induced in S-phase and show defects in recovering from checkpoint activation, both suppressed by checkpoint-adaptor deficient alleles of MRC1 (Fong et al. 2013). It was thus proposed that Mrc1 degradation through Dia2-mediated ubiquitylation is important to achieve checkpoint inactivation. Of note, Dia2 itself is ubiquitiylated and degraded via the proteasome in a mechanism counteracted by checkpoint kinases (Kile and Koepp 2010), thus hinting at the existence of a feedback loop promoting checkpoint inactivation by coupling stabilization of Dia2 and degradation of Mrc1. Mrc1 has also been described to interplay with the Rtt101 ${ }^{\mathrm{Mms} 22}$ ligase in response to replication stress in a pathway unrelated to Dia2 (Buser et al. 2016). Mutants impairing Rtt101 Mms22 function show sensitivity to the alkylating agent methylmethan sulphonate (MMS), which is suppressed by MRC1 deletion, but not by alleles abrogating its checkpoint mediator function. Rtt101 ${ }^{\mathrm{Mms} 22}$ is recruited to replication forks via the Ctf4 replisome component and does not appear to affect Mrc1 stability. These data suggest that in response to MMS-induced damage Mrc1 imposes a requirement for a yet unknown Rtt101 ${ }^{\mathrm{Mms} 22}$ function, perhaps in preserving replication fork integrity. Alternatively, Rtt101 ${ }^{\mathrm{Mms} 22}$ may counteract a 
yet not characterized function of Mrc1 in promoting DNA replication that may result deleterious in the presence of a damaged template. In human cells, CLASPIN is ubiquitylated by the $\mathrm{APC} / \mathrm{C}^{\mathrm{Cdh} 1}$ ubiquitin ligase complex, which promotes CLASPIN degradation via the proteasome both in the G1 phase of the cell cycle and in $\mathrm{G} 2 / \mathrm{M}$ during recovery from treatment with DNA damaging agents (Bassermann et al. 2008). CLASPIN ubiquitylation by $\mathrm{APC} / \mathrm{C}^{\mathrm{Cdh} 1}$ is counteracted by the USP28 ubiquitin protease. The $\mathrm{SCF}^{\beta \operatorname{TrCP}}$ ubiquitin ligase complex has also been involved in CLASPIN ubiquitylation upon entry in mitosis following recovery from genotoxic stress (Mailand et al. 2006). This modification has been proposed to contribute to checkpoint inactivation by limiting CLASPIN function as an adaptor and is counteracted by the ubiquitin protease USP7. Additionally, a recent work identified USP29 as an ubiquitin protease counteracting CLASPIN ubiquitylation and degradation (Martín et al. 2014). Down-regulation of USP29 function results in S-phase progression defects and impaired activation of the CHK1 kinase, though the ligase introducing the ubiquitin chains removed by USP29 remains to be elucidated. Lastly, a role in modulating CHK1 function through CLASPIN deubiquitylation has also been reported for the USP20 ubiquitin protease (Zhu et al. 2014). Collectively, this evidence points at a thigh control of Mrc1/CLASPIN function through ubiquitylation and degradation in the modulation of checkpoint signaling following replication stress.

It was recently shown that the ATR checkpoint response can negatively regulate ubiquitylation machineries. In particular, silencing of CHK1 destabilizes APC/CCdh1 targets in human cells experiencing replication blocks, in a fashion dependent on expression of the ligase (Yamada et al. 2013). It was thus suggested that the checkpoint kinase inhibits the ubiquitin ligase complex in a process required to stabilize the Dbf4/ASK component of the DDK complex and promote DNA damage bypass mechanisms. In consistence with this idea, chromatin loading of both the Rad18 ubiquitin ligase and Pol $\eta$ translesion polymerase is impaired by depletion of CHK1 upon induction of inter-strand crosslinks. Thus bidirectional crosstalk likely occurs between checkpoint and ubiquitylation machineries in the response to DNA lesions generating replication stress. 


\subsection{Ubiquitylation in the regulation of DNA polymerases association to DNA and chromatin dynamics.}

Ubiquitylation of DNA polymerases also appears to be important in the response to replication stress. In eukaryotes DNA polymerase $\varepsilon$ primarily carries out leading strand synthesis while DNA polymerase $\delta$ extends Okazaki fragments generated at lagging strands (Pursell et al. 2007). Ubiquitylation of the catalytic subunits of either replicative polymerase has been described in yeast cells (Roseaulin et al. 2013; Daraba et al. 2014). Inactivation of the Replication Fork Protection complex by ablation of Swi1/Tof1 in S. pombe cells, which presumably enhances replication stalling and collapse at natural fork pausing sites, leads to ubiquitylation by SCFPof3 and degradation of the Pol2 catalytic subunit of DNA polymerase $\varepsilon$ (Roseaulin et al. 2013). Ultraviolet (UV) light irradiation of S. cerevisiae cells induces DNA polymerase $\delta$ catalytic subunit Pol3 ubiquitylation and proteasomal degradation in a fashion dependent on Def1, a factor also involved in RNA polymerase II degradation (Daraba et al. 2014). Sensitivity of def1 mutants to MMS treatment is epistatic to that conferred by genes required for the error-free pathway of DNA damage tolerance, which raised the possibility that degradation might facilitate exchange of Pol3 by translesion synthesis polymerases. In agreement with this hypothesis DNA polymerase $\delta$ auxiliary subunits Pol31 and Pol32 are not degraded in these conditions and form a complex with DNA polymerase $\zeta$ associated Rev1 protein, while Def1 ablation reduces UV-induced mutation rates.

Ubiquitylation has been shown to mediate the extraction of translesion polymerases from DNA by the action of the Cdc48/p97 cofactor DVC1/SPARTAN (Mosbech et al. 2012). DVC1 is recruited to sites of stalled replication in fashion dependent of its UBZ (ubiquitin-binding zinc finger) domain, which lead to the hypothesis that it might interact with ubiquitylated replisome components. Interference of DVC1 or expression of an ATPase-deficient version of p97 results in increased Pol $\eta$ interaction with PCNA in UV-irradiated cells. DVC1 also enhances p97 association to UV-damaged chromatin, which suggests that polyubiquitylation of Pol $\eta$ may promote engagement by p97 to mediate its extraction 
during the last steps of translesion synthesis. In addition to this mechanism, it has been described that $\mathrm{Pol} \eta$ is mono-ubiquitylated in unperturbed cells and that this modification decreases upon treatment with genotoxic agents (Bienko et al. 2010). Mono-ubiquitylation inhibits Pol $\eta$ interaction with PCNA, and in this way may limit the basal frequency of TLS events.

DVC1/SPARTAN, as its budding yeast paralog Wss1, bears a metalloprotease activity, which is dependent on its association to DNA and is involved in replication-dependent repair of DNA-protein crosslinks (DPC) (Stingele and Jentsch 2015; Stingele et al. 2016; Vaz et al. 2016). DVC1 in enriched at nascent DNA and interacts with replisome factors such as PCNA and the MCM complex, suggesting that it acts on DPCs as they are encountered by replication forks (Stingele et al. 2016; Vaz et al. 2016). Consistently, ablation of DVC1 in human or worm cells leads to lethality and fork progression defects upon treatment with agents inducing protein crosslinking to DNA. DVC1 is ubiquitylated and its deubiquitylation promotes its access to chromatin, perhaps by disengagement of in-cis interactions of its UBZ domain. Whether DVC1 function in replicationcoupled DPC repair involves its role as an adaptor for Cdc48/p97 in extraction ubiquitylated proteins from chromatin remains to be elucidated.

Ubiquitylation also plays a critical role in regulating replication in the chromatin context. Polycomb repressive complexes (PRCs) mediate the establishment of repressive chromatin through ubiquitylation of H2A (Di Croce and Helin 2013). In addition, H2A ubiquitylation by PCRs has recently emerged as a regulator of DNA replication. PRCs associate to nascent DNA in mouse cells and interference of their function impairs replication fork progression in the absence of external perturbations (Piunti et al. 2014). These observations suggest that PCRs may modulate chromatin dynamics in particular chromosome territories to facilitate fork progression. Consistently, inactivation of RING ubiquitin ligase activities associated to PCRs leads S-phase defects and increased asymmetry in replication fork progression (Bravo et al. 2015). Replication impairment in these conditions is specific to pericentromeric heterochromatic (PCH) domains and can be suppressed by targeted restoration of $\mathrm{H} 2 \mathrm{~A}$ ubiquitylation at $\mathrm{PCH}$. The exact 
function of $\mathrm{H} 2 \mathrm{~A}$ ubiquitylation during replication remains to be understood, but might reflect a necessity to modulate chromatin structure at hard-to-replicate domains or limit fork conflicts with de-repressed transcription.

H2B is mono-ubiquitylated by Bre1/RNF20 E3 ubiquitin ligase and this modification plays a well-characterized role in transcriptional regulation (Meas and Mao 2015). In budding yeast, Bre1 and mono-ubiquitylated H2B are enriched around active replication origins, suggesting that Bre1 associates to replication forks to promote H2B-ubiquitylation at nascent chromatin DNA (Trujillo and Osley 2012; Lin et al. 2014). Non-ubiquitylatable lysine-123 H2B mutants are sensitive to HU treatment; and show altered stalled fork progression as well as defects in recovery from replication stress, likely independent of transcriptional regulation of dNTP pools. It was thus suggested that H2B ubiquitylation is important coupling chromatin assembly and replication fork progression.

\subsection{Modulation of the ubiquitylation of the sliding clamp PCNA as a central regulator of DNA damage tolerance mechanisms.}

At the core of replicating chromatin PCNA works as an essential processivity factor for DNA polymerases (Moldovan et al. 2007; Ulrich 2009; Mailand et al. 2013). PCNA interacts with a number of proteins involved in replication itself as well as with factors involved in the maintenance of genome integrity (Mailand et al. 2013). The post-translational modification of PCNA licenses a layer of control in PCNAprotein interactions that facilitates fork progression under normal or perturbed replication (Andersen et al. 2008; Ulrich 2009; Bergink and Jentsch 2009; Mailand et al. 2013). During S-phase, cells face damaged DNA or lesions that pose significant barriers to the progression of replication forks (Curtin 2012). In living organisms, tolerance mechanisms ensure that DNA can be replicated when it is damaged (Friedberg 2003). These mechanisms prevent irreversible fork collapse when the replisome encounters bulky lesions at damaged sites that block replicative DNA polymerases (Friedberg et al. 2005). Cells have evolved tolerance 
to DNA lesions to ensure progression of replication forks past unrepaired damage (Lawrence 1994; Chang and Cimprich 2009).

Tolerance to DNA damage is based either on translesion synthesis (TLS) that is carried out by specialized low-fidelity, potentially error-prone, TLS DNA polymerases or on template switching, an error-free mechanism that involves sister-strand pairing within or nearby the replication fork. In the face of a DNA lesion, the ubiquitylation of the sliding clamp controls the choice of translesion synthesis in eukaryotes. While mono-ubiquitylation of PCNA at Lys ${ }^{164}$ enhances the affinity of error-prone TLS DNA polymerases (Hoege et al. 2002; Stelter and Ulrich 2003; Watanabe et al. 2004 ), further Lys ${ }^{63}$-linked poly-ubiquitylation of mono-ubiquitylated Lys ${ }^{164}$-PCNA promotes template switching (Zhang and Lawrence 2005; Vanoli et al. 2010). This control mechanism is well characterized and widely conserved in eukaryotic organisms (Moldovan et al. 2007; Ulrich 2009; Mailand et al. 2013). On the contrary, the significance of the deubiquitylation of PCNA remains insufficiently understood.

The ubiquitylation of PCNA is catalysed by proteins in the Rad6 epistasis group (Hoege et al. 2002; Stelter and Ulrich 2003) (Figure 5). The Rad6 group includes genes required for post-replication repair in the budding yeast $S$. cerevisiae, and consists of RAD6 and RAD18 (Lys ${ }^{164}$-PCNA-ubiquitin ligase) or RAD5, MMS2 and UBC13 (Lys ${ }^{63}$-linked Lys ${ }^{164}$-PCNA-ubiquitin ligase) (Lawrence 1994; Zhang and Lawrence 2005). The Rad6 epistasis group is a central player in DNA damage bypass and tolerance pathways (historically known as post-replication repair) as these enzyme-complexes are involved in protein ubiquitylation of PCNA and translesion synthesis DNA polymerases. PCNA monoubiquitylation is regarded as a central step in translesion synthesis during normal or perturbed DNA replication. However, DT40 avian cell line evidence indicates that Pol $\eta$ (a TLS-DNA polymerase) and PCNA interact in the absence of PCNA ubiquitylation (Edmunds et al. 2008). Furthermore, there is general consensus that PCNA ubiquitylation is important but not essential for translesion DNA synthesis in mammalian cells (Edmunds et al. 2008; Hendel et al. 2011). Nevertheless, all eukaryotic models tested to date monoubiquitylate Lys ${ }^{164}$-PCNA to detectable levels in response to 
replication blocks or DNA damage to direct TLS polymerases to damaged sites (Hoege et al. 2002; Kannouche et al. 2004; Watanabe et al. 2004; Arakawa et al. 2006; Frampton et al. 2006). Of particular interest is the fact that PCNA is monoubiquitylated in Xenopus and S. pombe, and di-ubiquitylated in S. pombe, during unperturbed S-phase in a Rad6/Rad18-dependent manner (Leach and Michael 2005; Frampton et al. 2006).

Rad18 is the predominant PCNA E3 ubiquitin-ligase in eukaryotes. However, evidence from mammalian cells indicate that other ubiquitin-ligases, including RNF8, HLTF and CRL4CDT2, may have a role in this process in the absence of Rad18 or during unperturbed DNA replication (Zhang et al. 2008; Terai et al. 2010; Lin et al. 2011). The choice between the two branches of damage tolerance, error-prone or error-free, relies respectively on the mono-ubiquitylation and polyubiquitylation of the sliding clamp PCNA. However, the timing of PCNA ubiquitylation, and therefore the timing of lesion bypass, relative to DNA replication remains under discussion. It has been shown that tolerance to DNA damage mediated by ubiquitin-dependent modification of PCNA can be uncoupled from bulk DNA replication, at least in S. cerevisiae (Karras and Jentsch 2010; Daigaku et al. 2010), suggesting the hypothesis that DNA damage bypass may work exclusively as a post-replicative mechanism (Karras and Jentsch 2010). It is worth to mention that PCNA-ubiquitin ligase Rad18 associates with replisomes at sites of newly synthesized DNA in human cells (Dungrawala et al. 2015; Despras et al. 2016), indicating that the machinery for ubiquitylation of PCNA travels with the replisome at replication forks.

Along the last decade mammalian USP1, human Usp10, and budding yeast Ubp10 have been identified as major deubiquitylating enzymes (DUBs) for PCNA (Huang et al. 2006; Gallego-Sánchez et al. 2012; Mailand et al. 2013; Park et al. 2014). USP1, the first PCNA-DUB identified, is a ubiquitin protease that deubiquitylates monoubPCNA and mono-ubFANCD2 in human cells (Huang et al. 2006). USP1 undergoes inactivation by auto-proteolysis when cells are irradiated with UV light. Therefore, upon UV-irradiation USP1 is inactivated and PCNA becomes ubiquitylated, this observation suggest that USP1 continuously deubiquitylates PCNA in the absence 
of DNA damage. Consistent with a role as a PCNA-DUB, the depletion of chicken USP1 in DT40 cells or in murine USP1-/- MEFs increases PCNA, and also FANCD2, mono-ubiquitylation (Oestergaard et al. 2007; Kim et al. 2009). Recently, it has been shown that upon UV-mediated DNA damage, HeLa cells rely on USP10 to deubiquitylate ISGylated-PCNA (Park et al. 2014). Two unicellular model systems have been examined in detail, budding and fission yeast. In the budding yeast $S$. cerevisiae, the ubiquitin protease Ubp10 deubiquitylates Lys ${ }^{164}$ mono-and diubiquitylated PCNA during S phase (Gallego-Sánchez et al. 2012, 2013). More recently, it has been shown that Ubp2, Ubp15, Ubp16, and Ubp12 ubiquitin proteases revert PCNA ubiquitylation in the fission yeast $S$. pombe during S-phase (Álvarez et al. 2016). To date, all the identified PCNA-DUBs belong to the USP/UBP subfamily of ubiquitin-specific proteases.

All the published evidence provides robust evidence that PCNA-DUBs revert the Lys ${ }^{164}$ mono- and di-ubiquitylation of PCNA in vitro and in vivo. Therefore, PCNADUBs activities may counterbalance the ubiquitin ligase activity of the Rad6/Rad18 complex responsible for PCNA mono-ubiquitylation at sites of newly synthesized DNA. Thus, these ubiquitin-specific proteases have the potential to be part of a safeguard mechanism limiting the residence time of TLS DNA polymerases on replicating chromatin (Figure 5).

\subsection{Ubiquitylation-mediated control of the Fanconi Anemia DNA repair pathway.}

Fanconi anemia (FA) is a rare disorder characterized by sensitivity to DNA interstrand crosslinking agents, bone-marrow failure and susceptibility to both acute myeloid leukemia and solid tumors. These features can also be accompanied by congenital abnormalities and infertility (Risitano et al. 2016). The Fanconi Anemia pathway (named after the syndrome) responds to DNA interstrand crosslinks, replication fork-stalling lesions that hamper not only the DNA synthesis by polymerases but also strand separation by replicative helicases. The FA pathway promotes the repair of DNA ICLs orchestrating several processes that 
include recognition of the ICL (Figure 6), incision of the lesion by structurespecific endonucleases, bypass by TLS polymerases and HR-mediated repair of the incised strand. The pathway is initiated by the recruitment of the core FA complex, which contains the E3 ubiquitin ligase FANCL, to ICLs upon engagement by replication forks (Longerich et al. 2009). Unloading of the CMG helicase complex from chromatin, a step required for the last steps of DNA synthesis and subsequent nucleolytic incision, follows ICL recognition. CMG extraction requires polyubiquitylation of MCM7 that is mediated by BRCA1 E3 ligase, in contrast with replication termination in which is mediated by $\mathrm{SCF}^{\mathrm{Dia} 2}$, which promotes engagement by the Cdc48/p97segregase (Long et al. 2014; Fullbright et al. 2016). Upon recruitment to stalled forks the catalytic subunit FANCL monoubiquitylates the FANCD2-FANCI dimer. This ubiquitylation activates downstream events leading to lesion processing and repair. How these events are coordinated to achieve ICL removal starts to be elucidated, as some key mechanistic details have been recently described. Ubiquitylation of FANCD2 is essential for its interaction with a UBZ4 domain in the SLX4 scaffold protein. SLX4 in turn promotes recruitment and activation of several nucleases, such as ERCC4-ERCC1, MUS81EME1 and SLX1, which mediate the incisions of a DNA strand flanking the ICL. Ubiquitylated FANCD2 also mediates its interaction with the UBZ domain of FAN1 (Fanconi Anemia Nuclease 1), yet another nuclease involved in the FA-mediated repair (Liu et al. 2010; Yoshikiyo et al. 2010). FAN1 is dispensable for the incision of ICLs in vitro and FAN1 mutations are not associated to typical FA phenotypes (Klein Douwel et al. 2014), suggesting that it may mediate FA-pathway functions unrelated to ICL repair. Accordingly, FANCD2 recruits FAN1 to replication forks stalled by HU treatment where is required for efficient restart (Lachaud et al. 2016). Of note, FANCD2 patient-derived cells exhibit increased replication fork stalling at common fragile sites (CFS) (Madireddy et al. 2016), suggesting that FANCD2 ubiquitylation may serve as a signal mediating distinct fork protection mechanisms besides the repair of ICLs.

There is evidence of crosstalk between the FA-pathway and key regulators of the DNA damage tolerance mechanisms. As mentioned above, Rad18 mediates PCNA mono-ubiquitylation and is also required for the ubiquitylation and activation of 
FANCD2/I (Geng et al. 2010; Williams et al. 2011). However, how Rad18 contributes to activation of the FA pathway is a matter of debate. On the one hand, it was proposed that FANCL interacts with ubiquitylated-PCNA and that this interaction is necessary for recruitment of FANCL to chromatin and FANCD2 mono-ubiquitylation (Geng et al. 2010). On the other hand, it has been reported that Rad18 promotes FACND2/I ubiquitylation in a fashion independent on PCNAK164 (Williams et al. 2011), in apparent contradiction with the previous report. In addition, it has been shown that FANCD2 interacts with PCNA through a PIP box and that FANCD2 PIP-box mutants show impaired ubiquitylation (Williams et al. 2011), though the involvement of Rad18 or PCNA mono-ubiquitylation in this mechanism was not explored. Another link between FA and DDT pathways occurs through the USP1 ubiquitin protease that, as mentioned, reverts both the monoubiquitylation of both PCNA and the FANCD2-I complex (Nijman et al. 2005a). Silencing of USP1 expression increases mutation frequencies in UV-treated cells (Nijman et al. 2005a), suggesting that a fine regulation of these two processes is important to promote replication fidelity. Inactivation of the FA pathway involves SUMOylation of FANCD2 and FANCI proteins by PIAS1 and PIAS4 SUMO E3 ligases (Gibbs-Seymour et al. 2015). SUMOylated FANCD2-I is engaged by the RNF4 SUMO-targeted ubiquitin ligase, which in turn polyubiquitylates FANCD2-I and promotes eviction of the dimer from chromatin. FANCD2-I is extracted by the p97 segregase with the aid of its adaptor DVC1, thus locally interrupting FA pathway signaling.

\section{Concluding remarks.}

In recent years our knowledge on the flexibility and complexity of the ubiquitylation code has rapidly expanded. This is reflected in the current understanding of how ubiquitylation dynamically regulates DNA replication. Ubiquitin-mediated degradation controls the stability of factors spatiotemporally regulating replication initiation, as well as proteins mediating replication stress signaling. Ubiquitylation of replication factors also modulates important DNA repair of DNA damage tolerance pathway choices in response to replication stress. Lastly, the importance of ubiquitylation as a signal to control the extraction of key 
proteins from replicating chromatin by ubiquitin-selective segregase complexes during termination and upon fork stalling is emerging. It is thus expected that research in years to come may elucidate key mechanistic details of how ubiquitin regulates DNA replication, replication fork protection and the coordination of replication with other chromosome metabolic processes.

\section{Acknowledgments.}

We apologize for relevant findings and studies that might have not been discussed due to space limitations. This work was supported by the Ministry of Economy and Competitiveness (BFU2014-52529-R to R.B. and BFU2015-69709-P A.B.). S.V-H received support from the Spanish Formación del Personal Investigador (FPI) program.

\section{References}

Alcasabas AA, Osborn AJ, Bachant J, et al (2001) Mrc1 transduces signals of DNA replication stress to activate Rad53. Nat Cell Biol 3:958-965. doi:

$10.1038 /$ ncb1101-958

Álvarez V, Viñas L, Gallego-Sánchez A, et al (2016) Orderly progression through Sphase requires dynamic ubiquitylation and deubiquitylation of PCNA. Sci Rep 6:25513. doi: $10.1038 /$ srep25513

Andersen PL, Xu F, Xiao W (2008) Eukaryotic DNA damage tolerance and translesion synthesis through covalent modifications of PCNA. Cell Res 18:162-173. doi: 10.1038/cr.2007.114

Arakawa H, Moldovan GL, Saribasak H, et al (2006) A role for PCNA ubiquitination in immunoglobulin hypermutation. PLoS Biol 4:1947-1956. doi:

10.1371/journal.pbio.0040366

Bassermann F, Frescas D, Guardavaccaro D, et al (2008) The Cdc14B-Cdh1-Plk1 Axis Controls the G2 DNA-Damage-Response Checkpoint. Cell 134:256-267. doi: 10.1016/j.cell.2008.05.043

Bell SP, Labib K (2016) Chromosome Duplication in Saccharomyces cerevisiae. Genetics 203:1027-1067. doi: 10.1534/genetics.115.186452 
Bell SP, Stillman B (1992) ATP-dependent recognition of eukaryotic origins of DNA replication by a multiprotein complex. Nature 357:128-34. doi: $10.1038 / 357128 \mathrm{a} 0$

Bergink S, Jentsch S (2009) Principles of ubiquitin and SUMO modifications in DNA repair. Nature 458:461-7. doi: 10.1038/nature07963

Bienko M, Green CM, Sabbioneda S, et al (2010) Regulation of Translesion Synthesis DNA Polymerase $\eta$ by Monoubiquitination. Mol Cell 37:396-407. doi: 10.1016/j.molcel.2009.12.039

Böhm S, Bernstein KA (2014) The role of post-translational modifications in finetuning BLM helicase function during DNA repair. DNA Repair (Amst) 22:123132. doi: 10.1016/j.dnarep.2014.07.007

Branzei D, Foiani M (2010) Maintaining genome stability at the replication fork. Nat Rev Mol Cell Biol 11:208-19. doi: 10.1038/nrm2852

Bravo M, Nicolini F, Starowicz K, et al (2015) Polycomb RING1A- and RING1Bdependent histone H2A monoubiquitylation at pericentromeric regions promotes S-phase progression. J Cell Sci 128:3660-3671. doi: $10.1242 /$ jcs.173021

Bugreev D V, Yu X, Egelman EH, Mazin A V (2007) Novel pro- and antirecombination activities of the Bloom 's syndrome helicase. Genes Dev 21:3085-3094. doi: 10.1101/gad.1609007.cluding

Buser R, Kellner V, Melnik A, et al (2016) The Replisome-Coupled E3 Ubiquitin Ligase Rtt101Mms22 Counteracts Mrc1 Function to Tolerate Genotoxic Stress. PLOS Genet 12:e1005843. doi: 10.1371/journal.pgen.1005843

Carr AM, Lambert S (2013) Replication stress-induced genome instability: The dark side of replication maintenance by homologous recombination. J Mol Biol 425:4733-4744. doi: 10.1016/j.jmb.2013.04.023

Chang DJ, Cimprich KA (2009) DNA damage tolerance: when it's OK to make mistakes. Nat Chem Biol 5:82-90. doi: 10.1038/nchembio.139

Chu WK, Payne MJ, Beli P, et al (2015) FBH1 influences DNA replication fork stability and homologous recombination through ubiquitylation of RAD51. Nat Commun 6:1-9. doi: 10.1038/ncomms6931

Curtin NJ (2012) DNA repair dysregulation from cancer driver to therapeutic target. Nat Rev Cancer 12:801-817. doi: 10.1038/nrc3399 
Daigaku Y, Davies AA, Ulrich HD (2010) Ubiquitin-dependent DNA damage bypass is separable from genome replication. Nature 465:951-5. doi:

\section{$10.1038 /$ nature09097}

Daraba A, Gali VK, Halmai M, et al (2014) Def1 promotes the degradation of Pol3 for polymerase exchange to occur during DNA-damage--induced mutagenesis in Saccharomyces cerevisiae. PLoS Biol 12:e1001771. doi:

10.1371/journal.pbio.1001771

Despras E, Sittewelle M, Pouvelle C, et al (2016) Rad18-dependent SUMOylation of human specialized DNA polymerase eta is required to prevent underreplicated DNA. Nat Commun 7:13326. doi: 10.1038/ncomms13326

Dewar JM, Low E, Mann M, et al (2017) CRL2 Lrr1 promotes unloading of the vertebrate replisome from chromatin during replication termination. Genes Dev 275-290. doi: 10.1101/gad.291799.116

Di Croce L, Helin K (2013) Transcriptional regulation by Polycomb group proteins. Nat Struct Mol Biol 20:1147-55. doi: 10.1038/nsmb.2669

Diffley JFX, Cocker JH, Dowell SJ, Rowley A (1994) Two steps in the assembly of complexes at yeast replication origins in vivo. Cell 78:303-316. doi: 10.1016/0092-8674(94)90299-2

Donzelli M, Squatrito M, Ganoth D, et al (2002) Dual mode of degradation of Cdc25 A phosphatase. EMBO J 21:4875-4884. doi: 10.1093/emboj/cdf491

Dungrawala H, Rose KLL, Bhat KPP, et al (2015) The Replication Checkpoint Prevents Two Types of Fork Collapse without Regulating Replisome Stability. Mol Cell 59:1-13. doi: 10.1016/j.molcel.2015.07.030

Edmunds CE, Simpson LJ, Sale JE (2008) PCNA Ubiquitination and REV1 Define Temporally Distinct Mechanisms for Controlling Translesion Synthesis in the Avian Cell Line DT40. Mol Cell 30:519-529. doi: 10.1016/j.molcel.2008.03.024 Elia AEH, Wang DC, Willis NA, et al (2015) RFWD3-Dependent Ubiquitination of RPA Regulates Repair at Stalled Replication Forks. Mol Cell 60:280-293. doi: 10.1016/j.molcel.2015.09.011

Fong CM, Arumugam A, Koepp DM (2013) The Saccharomyces cerevisiae F-box protein Dia2 is a mediator of S-phase checkpoint recovery from DNA damage. Genetics 193:483-99. doi: 10.1534/genetics.112.146373

Frampton J, Irmisch A, Green CM, et al (2006) Postreplication Repair and PCNA 
Modification in Schizosaccharomyces pombe. 17:2976-2985. doi:

10.1091/mbc.E05

Francis LI, Randell JCW, Takara TJ, et al (2009) Incorporation into the prereplicative complex activates the Mcm2 - 7 helicase for Cdc7 - Dbf4 phosphorylation. Genes Dev 23:643-654. doi:

10.1101/gad.1759609.replication

Franz A, Orth M, Pirson PA, et al (2011) CDC-48/p97 Coordinates CDT-1 Degradation with GINS Chromatin Dissociation to Ensure Faithful DNA Replication. Mol Cell 44:85-96. doi: 10.1016/j.molcel.2011.08.028

Friedberg EC (2003) DNA damage and Repair.

Friedberg EC, Lehmann AR, Fuchs RPP (2005) Trading places: how do DNA polymerases switch during translesion DNA synthesis? Mol Cell 18:499-505. doi: 10.1016/j.molcel.2005.03.032

Fullbright G, Rycenga HB, Gruber JD, Long DT (2016) p97 Promotes a Conserved Mechanism of Helicase Unloading During DNA Crosslink Repair. Mol Cell Biol MCB.00434-16. doi: 10.1128/MCB.00434-16

Gaillard H, García-Muse T, Aguilera A (2015) Replication stress and cancer. Nat Rev Cancer 15:276-289. doi: 10.1038/nrc3916

Gallego-Sánchez A, Andrés S, Conde F, et al (2012) Reversal of PCNA ubiquitylation by Ubp10 in Saccharomyces cerevisiae. PLoS Genet. doi:

10.1371/journal.pgen.1002826

Gallego-Sánchez A, Andrés S, Ufano S, Bueno A (2013) Analysis of the Tolerance to DNA Alkylating Damage in MEC1 and RAD53 Checkpoint Mutants of Saccharomyces cerevisiae. PLoS One 8:e81108. doi:

10.1371/journal.pone.0081108

Geng L, Huntoon CJ, Karnitz LM (2010) RAD18-mediated ubiquitination of PCNA activates the Fanconi anemia DNA repair network. J Cell Biol 191:249-257. doi: $10.1083 / j c b .201005101$

Ghospurkar PL, Wilson TM, Severson a. L, et al (2015) The DNA Damage Response and Checkpoint Adaptation in Saccharomyces cerevisiae: Distinct Roles for the Replication Protein A2 (Rfa2) N-Terminus. Genetics 199:711-727. doi: 10.1534/genetics.114.173211

Gibbs-Seymour I, Oka Y, Rajendra E, et al (2015) Ubiquitin-SUMO circuitry controls 
activated fanconi anemia ID complex dosage in response to DNA damage. Mol Cell 57:150-164. doi: 10.1016/j.molcel.2014.12.001

Hendel A, Krijger PHL, Diamant N, et al (2011) PCNA ubiquitination is important, but not essential for translesion DNA synthesis in mammalian cells. PLoS Genet 7:e1002262. doi: 10.1371/journal.pgen.1002262

Hernández-Pérez S, Cabrera E, Amoedo H, et al (2016) USP37 deubiquitinates Cdt1 and contributes to regulate DNA replication. Mol Oncol 0:1-11. doi: 10.1016/j.molonc.2016.05.008

Hoege C, Pfander B, Moldovan G-L, et al (2002) RAD6-dependent DNA repair is linked to modification of PCNA by ubiquitin and SUMO. Nature 419:135-141. doi: 10.1038/nature00991

Huang TT, Nijman SMB, Mirchandani KD, et al (2006) Regulation of monoubiquitinated PCNA by DUB autocleavage. Nat Cell Biol 8:339-47. doi: $10.1038 /$ ncb1378

Ilves I, Petojevic T, Pesavento JJ, Botchan MR (2010) Activation of the MCM2-7 Helicase by Association with Cdc45 and GINS Proteins. Mol Cell 37:247-258. doi: 10.1016/j.molcel.2009.12.030

Iwahori S, Kohmon D, Kobayashi J, et al (2014) ATM regulates Cdt1 stability during the unperturbed S phase to prevent re-replication. 7:471-481.

Jossen R, Bermejo R (2013) The DNA damage checkpoint response to replication stress: A Game of Forks. Front Genet 4:26. doi: 10.3389/fgene.2013.00026

Kannouche PL, Wing J, Lehmann AR (2004) Interaction of human DNA polymerase eta with monoubiquitinated PCNA: A possible mechanism for the polymerase switch in response to DNA damage. Mol Cell 14:491-500. doi: 10.1016/S1097-2765(04)00259-X

Karras GI, Jentsch S (2010) The RAD6 DNA damage tolerance pathway operates uncoupled from the replication fork and is functional beyond $S$ phase. Cell 141:255-67. doi: 10.1016/j.cell.2010.02.028

Kee Y, Lyon N, Huibregtse JM (2005) The Rsp5 ubiquitin ligase is coupled to and antagonized by the Ubp2 deubiquitinating enzyme. EMBO J 24:2414-24. doi: 10.1038/sj.emboj.7600710

Kile AC, Koepp DM (2010) Activation of the S-phase checkpoint inhibits degradation of the F-box protein Dia2. Mol Cell Biol 30:160-71. doi: 
10.1128/MCB.00612-09

Kim JM, Parmar K, Huang M, et al (2009) Inactivation of Murine Usp1 Results in Genomic Instability and a Fanconi Anemia Phenotype. Dev Cell 16:314-320. doi: 10.1016/j.devcel.2009.01.001

Klein Douwel D, Boonen RACM, Long DT, et al (2014) XPF-ERCC1 Acts in Unhooking DNA Interstrand Crosslinks in Cooperation with FANCD2 and FANCP/SLX4. Mol Cell 54:460-471. doi: 10.1016/j.molcel.2014.03.015

Komander D, Rape M (2012) The Ubiquitin Code. Annu Rev Biochem 81:203-229. doi: 10.1146/annurev-biochem-060310-170328

Kulathu Y, Komander D (2012) Atypical ubiquitylation - the unexplored world of polyubiquitin beyond Lys48 and Lys63 linkages. Nat Rev Mol Cell Biol 13:508-23. doi: 10.1038/nrm3394

Lachaud C, Moreno A, Marchesi F, et al (2016) Ubiquitinated Fancd2 recruits Fan1 to stalled replication forks to prevent genome instability. Science (80-) 351:846-849. doi: 10.1126/science.aad5634

Lawrence C (1994) The RAD6 DNA repair pathway in Saccharomyces cerevisiae: What does it do, and how does it do it? BioEssays 16:253-258. doi: 10.1002/bies.950160408

Leach CA, Michael WM (2005) Ubiquitin/SUMO modification of PCNA promotes replication fork progression in Xenopus laevis egg extracts. J Cell Biol 171:947-954. doi: 10.1083/jcb.200508100

Lecona E, Rodriguez-Acebes S, Specks J, et al (2016) USP7 is a SUMO deubiquitinase essential for DNA replication. Nat Struct Mol Biol. doi: 10.1038/nsmb.3185

Li X, Zhao Q, Liao R, et al (2003) The SCFSkp2 ubiquitin ligase complex interacts with the human replication licensing factor Cdt1 and regulates Cdt1 degradation. J Biol Chem 278:30854-30858. doi: 10.1074/jbc.C300251200

Lin C-Y, Wu M-Y, Gay S, et al (2014) H2B Mono-ubiquitylation Facilitates Fork Stalling and Recovery during Replication Stress by Coordinating Rad53 Activation and Chromatin Assembly. PLoS Genet 10:e1004667. doi: 10.1371/journal.pgen.1004667

Lin JR, Zeman MK, Chen JY, et al (2011) SHPRH and HLTF Act in a Damage-Specific Manner to Coordinate Different Forms of Postreplication Repair and Prevent 
Mutagenesis. Mol Cell 42:237-249. doi: 10.1016/j.molcel.2011.02.026

Liu T, Ghosal G, Yuan J, et al (2010) FAN1 acts with FANCI-FANCD2 to promote DNA interstrand cross-link repair. Science 329:693-696. doi:

10.1126/science.1192656

Long DT, Joukov V, Budzowska M, Walter JC (2014) BRCA1 Promotes Unloading of the CMG Helicase from a Stalled DNA Replication Fork. Mol Cell 1-12. doi: 10.1016/j.molcel.2014.08.012

Longerich S, San Filippo J, Liu D, Sung P (2009) FANCI binds branched DNA and is monoubiquitinated by UBE2T-FANCL. J Biol Chem 284:23182-23186. doi: 10.1074/jbc.C109.038075

Maculins T, Nkosi PJ, Nishikawa H, Labib K (2015) Tethering of SCFDia2 to the Replisome Promotes Efficient Ubiquitylation and Disassembly of the CMG Helicase. Curr Biol 1-6. doi: 10.1016/j.cub.2015.07.012

Madireddy A, Kosiyatrakul ST, Boisvert RA, et al (2016) FANCD2 Facilitates Replication through Common Fragile Sites. Mol Cell 64:388-404. doi: 10.1016/j.molcel.2016.09.017

Mailand N, Bekker-Jensen S, Bartek J, Lukas J (2006) Destruction of Claspin by SCFbetaTrCP restrains Chk1 activation and facilitates recovery from genotoxic stress. Mol Cell 23:307-18. doi: 10.1016/j.molcel.2006.06.016

Mailand N, Gibbs-seymour I, Bekker-Jensen S (2013) Regulation of PCNA-protein interactions for genome stability. Nat Rev Mol Cell Biol 14:269-82. doi: $10.1038 / \mathrm{nrm} 3562$

Maréchal A, Li J-M, Ji XY, et al (2013) PRP19 Transforms into a Sensor of RPAssDNA after DNA Damage and Drives ATR Activation via a Ubiquitin-Mediated Circuitry. Mol Cell 53:1-12. doi: 10.1016/j.molcel.2013.11.002

Maric M, Maculins T, De Piccoli G, Labib K (2014) Cdc48 and a ubiquitin ligase drive disassembly of the CMG helicase at the end of DNA replication. Science (80- ) 346:1253596-1253596. doi: 10.1126/science.1253596

Martín Y, Cabrera E, Amoedo H, Freire R (2014) USP29 controls the stability of checkpoint adaptor Claspin by deubiquitination. 28:1-6. doi: 10.1038/onc.2014.38

McGarry TJ, Kirschner MW (1998) Geminin, an inhibitor of DNA replication, is degraded during mitosis. Cell 93:1043-1053. doi: 10.1016/S0092- 
8674(00)81209-X

Meas R, Mao P (2015) Histone ubiquitylation and its roles in transcription and DNA damage response. DNA Repair (Amst) 36:36-42. doi:

10.1016/j.dnarep.2015.09.016

Mimura S, Komata M, Kishi T, et al (2009) SCF(Dia2) regulates DNA replication forks during S-phase in budding yeast. EMBO J 28:3693-705. doi:

10.1038/emboj.2009.320

Moldovan G-L, Pfander B, Jentsch S (2007) PCNA, the maestro of the replication fork. Cell 129:665-79. doi: 10.1016/j.cell.2007.05.003

Mosbech A, Gibbs-Seymour I, Kagias K, et al (2012) DVC1 (C1orf124) is a DNA damage-targeting p97 adaptor that promotes ubiquitin-dependent responses to replication blocks. Nat Struct Mol Biol 19:1084-1092. doi:

10.1038/nsmb.2395

Muramatsu S, Hirai K, Tak YS, et al (2010) CDK-dependent complex formation between replication proteins Dpb11, Sld2, Pol ??, and GINS in budding yeast. Genes Dev 24:602-612. doi: 10.1101/gad.1883410

Newton K, Matsumoto ML, Wertz IE, et al (2008) Ubiquitin Chain Editing Revealed by Polyubiquitin Linkage-Specific Antibodies. Cell 134:668-678. doi:

10.1016/j.cell.2008.07.039

Nijman SMB, Huang TT, Dirac AMG, et al (2005a) The deubiquitinating enzyme USP1 regulates the fanconi anemia pathway. Mol Cell 17:331-339. doi:

10.1016/j.molcel.2005.01.008

Nijman SMBB, Luna-Vargas MPA a, Velds A, et al (2005b) A genomic and functional inventory of deubiquitinating enzymes. Cell 123:773-786. doi:

10.1016/j.cell.2005.11.007

Nishitani H, Sugimoto N, Roukos V, et al (2006) Two E3 ubiquitin ligases, SCF-Skp2 and DDB1-Cul4, target human Cdt1 for proteolysis. EMBO J 25:1126-1136. doi: 10.1038/sj.emboj.7601002

Oestergaard VH, Langevin F, Kuiken HJ, et al (2007) Deubiquitination of FANCD2 Is Required for DNA Crosslink Repair. Mol Cell 28:798-809. doi:

10.1016/j.molcel.2007.09.020

Park JM, Yang SW, Yu KR, et al (2014) Modification of PCNA by ISG15 Plays a Crucial Role in Termination of Error-Prone Translesion DNA Synthesis. Mol 
Cell 54:626-638. doi: 10.1016/j.molcel.2014.03.031

Pellicioli A, Foiani M (2005) Signal transduction: How Rad53 kinase is activated. Curr Biol 15:769-771. doi: 10.1016/j.cub.2005.08.057

Pinder JB, Attwood KM, Dellaire G (2013) Reading, writing, and repair: The role of ubiquitin and the ubiquitin-like proteins in DNA damage signaling and repair. Front Genet 4:1-14. doi: 10.3389/fgene.2013.00045

Piunti A, Rossi A, Cerutti A, et al (2014) Polycomb proteins control proliferation and transformation independently of cell cycle checkpoints by regulating DNA replication. Nat Commun 5:3649. doi: 10.1038/ncomms4649

Priego Moreno S, Bailey R, Campion N, et al (2014) Polyubiquitylation drives replisome disassembly at the termination of DNA replication. Science (80- ). doi: 10.1126/science.1253585

Pursell ZF, Isoz I, Lundström E-B, et al (2007) Yeast DNA Polymerase e Participates in Leading-Strand DNA Replication. Science (80- ) 317:127-130.

Quan Y, Xia Y, Liu L, et al (2015) Cell-Cycle-Regulated Interaction between Mcm10 and Double Hexameric Mcm2-7 Is Required for Helicase Splitting and Activation during S Phase. Cell Rep 13:2576-2586. doi: 10.1016/j.celrep.2015.11.018

Randell JCW, Fan A, Chan C, et al (2010) Mec1 Is One of Multiple Kinases that Prime the Mcm2-7 Helicase for Phosphorylation by Cdc7. Mol Cell 40:353363. doi: 10.1016/j.molcel.2010.10.017

Risitano AM, Marotta S, Calzone R, et al (2016) Twenty years of the Italian Fanconi Anemia Registry: Where we stand and what remains to be learned. Haematologica 101:319-327. doi: 10.3324/haematol.2015.133520

Roseaulin LC, Noguchi C, Martinez E, et al (2013) Coordinated Degradation of Replisome Components Ensures Genome Stability upon Replication Stress in the Absence of the Replication Fork Protection Complex. PLoS Genet 9:21-28. doi: 10.1371/journal.pgen.1003213

Sahtoe DD, Sixma TK (2015) Layers of DUB regulation. Trends Biochem Sci 1-12. doi: 10.1016/j.tibs.2015.05.002

Sakaguchi H, Takami T, Yasutani Y, et al (2012) Checkpoint Kinase ATR Phosphorylates Cdt2, a Substrate Receptor of CRL4 Ubiquitin Ligase, and Promotes the Degradation of Cdt1 following UV Irradiation. PLoS One. doi: 
10.1371/journal.pone.0046480

Sánchez M, Calzada A, Bueno A (1999) The Cdc6 protein is ubiquitinated in vivo for proteolysis in Saccharomyces cerevisiae. J Biol Chem 274:9092-9097. doi: 10.1074/jbc.274.13.9092

Shibata Y, Oyama M, Kozuka-Hata H, et al (2012) p47 negatively regulates IKK activation by inducing the lysosomal degradation of polyubiquitinated NEMO. Nat Commun 3:1061. doi: 10.1038/ncomms2068

Siddiqui K, On KF, Diffley JFX (2013) Regulating DNA replication in eukarya. Cold Spring Harb Perspect Biol 5:1-17. doi: 10.1101/cshperspect.a012922 Simandlova J, Zagelbaum J, Payne MJ, et al (2013) FBH1 helicase disrupts RAD51 filaments in vitro and modulates homologous recombination in mammalian cells. J Biol Chem 288:34168-34180. doi: 10.1074/jbcM113.484493

Sowa ME, Bennett EJ, Gygi SP, Harper JW (2009) Defining the Human Deubiquitinating Enzyme Interaction Landscape. Cell 138:389-403. doi: 10.1016/j.cell.2009.04.042

Stelter P, Ulrich HD (2003) Control of spontaneous and damage-induced mutagenesis by SUMO and ubiquitin conjugation. Nature 425:188-91. doi: 10.1038/nature01965

Stingele J, Bellelli R, Alte F, et al (2016) Mechanism and Regulation of DNA-Protein Crosslink Repair by the DNA-Dependent Metalloprotease SPRTN. Mol Cell 64:688-703. doi: 10.1016/j.molcel.2016.09.031

Stingele J, Jentsch S (2015) DNA-protein crosslink repair. Nat Rev Mol Cell Biol 16:455-460. doi: 10.1038/nrm4015

Sugimoto N, Tatsumi Y, Tsurumi T, et al (2004) Cdt1 Phosphorylation by Cyclin Adependent Kinases Negatively Regulates Its Function without Affecting Geminin Binding. J Biol Chem 279:19691-19697. doi:

10.1074/jbc.M313175200

Tanaka K, Russell P (2001) Mrc1 channels the DNA replication arrest signal to checkpoint kinase Cds1. Nat Cell Biol 3:966-972. doi: 10.1038/ncb1101-966

Terai K, Abbas T, Jazaeri AA, Dutta A (2010) CRL4Cdt2 E3 Ubiquitin Ligase Monoubiquitinates PCNA to Promote Translesion DNA Synthesis. Mol Cell 37:143-149. doi: 10.1016/j.molcel.2009.12.018

Tikoo S, Madhavan V, Hussain M, et al (2013) Ubiquitin-dependent recruitment of 
the Bloom Syndrome helicase upon replication stress is required to suppress homologous recombination. EMBO J 32:1778-1792. doi:

10.1038/emboj.2013.117

Trujillo KM, Osley MA (2012) A Role for H2B Ubiquitylation in DNA Replication. Mol Cell 48:734-746. doi: 10.1016/j.molcel.2012.09.019

Ulrich HD (2009) Regulating post-translational modifications of the eukaryotic replication clamp PCNA. DNA Repair (Amst) 8:461-469. doi:

10.1016/j.dnarep.2009.01.006

Ulrich HD, Walden H (2010) Ubiquitin signalling in DNA replication and repair. Nat Rev Mol Cell Biol 11:479-89. doi: 10.1038/nrm2921

Vanoli F, Fumasoni M, Szakal B, et al (2010) Replication and recombination factors contributing to recombination-dependent bypass of DNA lesions by template switch. PLoS Genet. doi: 10.1371/journal.pgen.1001205

Vassin VM, Anantha RW, Sokolova E, et al (2009) Human RPA phosphorylation by ATR stimulates DNA synthesis and prevents ssDNA accumulation during DNAreplication stress. J Cell Sci 122:4070-4080. doi: 10.1242/jcs.053702

Vaz B, Popovic M, Newman JA, et al (2016) Metalloprotease SPRTN/DVC1 Orchestrates Replication-Coupled DNA-Protein Crosslink Repair. Mol Cell 0:137-151. doi: 10.1016/j.molcel.2016.09.032

Walter D, Hoffmann S, Komseli E-S, et al (2016) SCFCyclin F-dependent degradation of CDC6 suppresses DNA re-replication. Nat Commun 7:10530. doi: $10.1038 /$ ncomms10530

Watanabe K, Tateishi S, Kawasuji M, et al (2004) Rad18 guides poleta to replication stalling sites through physical interaction and PCNA monoubiquitination. EMBO J 23:3886-96. doi: 10.1038/sj.emboj.7600383

Williams SA, Longerich S, Sung P, et al (2011) The E3 ubiquitin ligase RAD18 regulates ubiquitylation and chromatin loading of FANCD2 and FANCI. Blood 117:5078-5087. doi: 10.1182/blood-2010-10-311761

Wohlschlegel JA, Dwyer BT, Dhar SK, et al (2000) Inhibition of Eukaryotic DNA Replication by Geminin Binding to Cdt1. Science (80- ) 290:2309-2312. doi: 10.1126/science.290.5500.2309

Yamada M, Watanabe K, Mistrik M, et al (2013) ATR-Chk1-APC/CCdh1-dependent stabilization of Cdc7-ASK (Dbf4) kinase is required for DNA lesion bypass 
under replication stress. Genes Dev 27:2459-72. doi:

$10.1101 /$ gad.224568.113

Yau R, Rape M (2016) The increasing complexity of the ubiquitin code. Nat Publ Gr 18:579-586. doi: 10.1038/ncb3358

Yim H, Park JW, Woo SU, et al (2013) Phosphorylation of Cdc6 at serine 74, but not at serine 106, drives translocation of Cdc6 to the cytoplasm. J Cell Physiol 228:1221-1228. doi: 10.1002/jcp.24275

Yoshikiyo K, Kratz K, Hirota K, et al (2010) KIAA1018/FAN1 nuclease protects cells against genomic instability induced by interstrand cross-linking agents. Proc Natl Acad Sci U S A 107:21553-7. doi: 10.1073/pnas.1011081107

Zhang H, Lawrence CW (2005) The error-free component of the RAD6/RAD18 DNA damage tolerance pathway of budding yeast employs sister-strand recombination. Proc Natl Acad Sci U S A 102:15954-15959. doi: $10.1073 /$ pnas.0504586102

Zhang S, Chea J, Meng X, et al (2008) PCNA is ubiquitinated by RNF8. Cell Cycle 7:3399-3404. doi: 10.4161/cc.7.21.6949

Zhu M, Zhao H, Liao J, Xu X (2014) HERC2/USP20 coordinates CHK1 activation by modulating CLASPIN stability. Nucleic Acids Res 1-8. doi: 10.1093/nar/gku978

Zou L, Elledge SJ (2003) Sensing DNA damage through ATRIP recognition of RPAssDNA complexes. Science 300:1542-8. doi: 10.1126/science.1083430

\section{Figure Captions}

Figure 1. The ubiquitin system. a) Mechanism of ubiquitin conjugation and deconjugation. Ubiquitin conjugation requires the activities of three factors: an E1 activating enzyme, an E2 conjugating enzyme and an E3 ubiquitin ligase (see text for details). Ubiquitylation can be reversed by ubiquitin proteases, also known as deubiquitinases (DUBs), which hydrolyze lysine-ubiquitin bonds and can remove single ubiquitin moieties or entire chains. b) Different types of ubiquitylation have been reported. Attachment of a single ubiquitin moiety in a given lysine (Kx) is referred to as monoubiquitylation, while conjugation of ubiquitin moieties to 
different lysines (Kx-z) results in multi-ubiquitylation. Polyubiquitylation occurs when multiple rounds of ubiquitin conjugation are preformed on a substrateconjugated ubiquitin. The lysine used to extend ubiquitin chains can be the same (Ki) along the chain, or vary (Ki-Kii) giving raise to mixed polyubiquitylation. In addition, more than one lysine on an ubiquitin moiety can be used to extend chains resulting in branched polyubiquitin chains. Linear polyubiquitin chains arise if the residue for chain elongation is the initial methionine on the ubiquitin moiety.

Figure 2. Ubiquitylation in the control of replication origin licensing. In G1 ORC binds origin DNA. Cdc6 and Cdt1 mediate the loading of the MCM2-7 helicase complex, in a process called origin licensing, and lead to the formation of prereplication complexes (pre-RC) determining potential replication initiation sites. During the G1-S transition, Cdc6 and Cdt1 are ubiquitylated and degraded in order to avoid loading of new MCM2-7 complexes to already replicated DNA and prevent re-replication. Origin firing involves the formation of a pre-initiation complex (pre-IC) that depends on the phosphorylation of several replication factors by DDK and CDK. DDK and CDK activities are also required to activate the replicative helicase and promote initial DNA unwinding.

\section{Figure 3. CMG ubiquitylation and extraction from DNA during replication} termination.

Upon replication fork convergence during replication termination, the SCF ${ }^{\text {Dia2 }}$ ubiquitin ligase polyubiquitylates the MCM7 subunit of the CMG replicative helicase. MCM7 ubiquitylation determines the disassembly and extraction of CMG helicase components, mediated by the Cdc48/p97 ubiquitin selective segregase, presumably to facilitate the last steps of DNA synthesis.

\section{Figure 4. Modulation of RPA/Rad51 function and homologous recombination} at stalled replication forks. (i) RPA ubiquitylation by RFWD3 upon replication stress induction promotes fork restart. Ubiquitylation likely promotes exchange of RPA by Rad51, thus promoting fork restart perhaps through recombination mediated mechanisms. (ii) Besides its ability to disassemble Rad51 filaments, the FBH1 helicase/E3 ligase ubiquitylates Rad51. Ubiquitylation of Rad51 likely 
promotes its eviction from DNA thus limiting toxic recombination impairing stalled fork functionality. (iii) RNF8 and RNF168 E3 ligases polyubiquitylate BLM in three different lysines. This modification is required for BLM recruitment to sites of stalled replication, where it is thought to suppress unscheduled recombination events by promoting the disruption of Rad51 nucleofilaments.

Figure 5. Model for PCNA ubiquitylation/deubiquitylation in the modulation of tolerance of DNA damage encountered by replication forks. PCNA progresses in association with the replisome on leading strands and associates to Pol $\delta$ on lagging strands. Bulky lesions block the progression of replicative polymerases and induce Rad6/Rad18-mediated PCNA monoubiquitylation. Ubiquitylated PCNA enhances the interaction with translesion synthesis DNA polymerases. Further Lys63-linked ubiquitylation of PCNA Lys164-conjugated ubiquitin by a second ubiquitin ligase complex (Ubc13/Mms2/Rad5) promotes template switching events. After lesion bypass, PCNA-DUBs deubiquitylate PCNA to switching back to replicative DNA polymerases and resume processive DNA replication. Dynamic ubiquitylation and deubiquitylation of PCNA may regulate DNA damage tolerance at replication forks and/or post-replicatively.

\section{Figure 6. Ubiquitylation is central in the modulation of the Fanconi Anemia} DNA repair pathway. Upon collision with replication forks the FANCM translocase recognizes interstrand crosslinks (ICL) and initiates the FA repair pathway. CMG ubiquitylation by BRCA1 E3 ligase drives its eviction through the action of the Cdc48/p97 segregase, which enables synthesis of nucleotides nearby the lesion. Then the Fanconi Anemia core complex is recruited to the lesion, where the FANCL ubiquitin ligase promotes the monoubiquitylation of the FANCD2/I heterodimer. This ubiquitylation is the central event that mediates the recruitment of the SLX4 protein, which serves a scaffold for nucleases that promote incisions on the DNA backbone around the lesion. DNA incision triggers downstream events in the FA pathway such as base flipping, translesion synthesis and HR-mediated repair of the incised strand. 
Figure 1

a
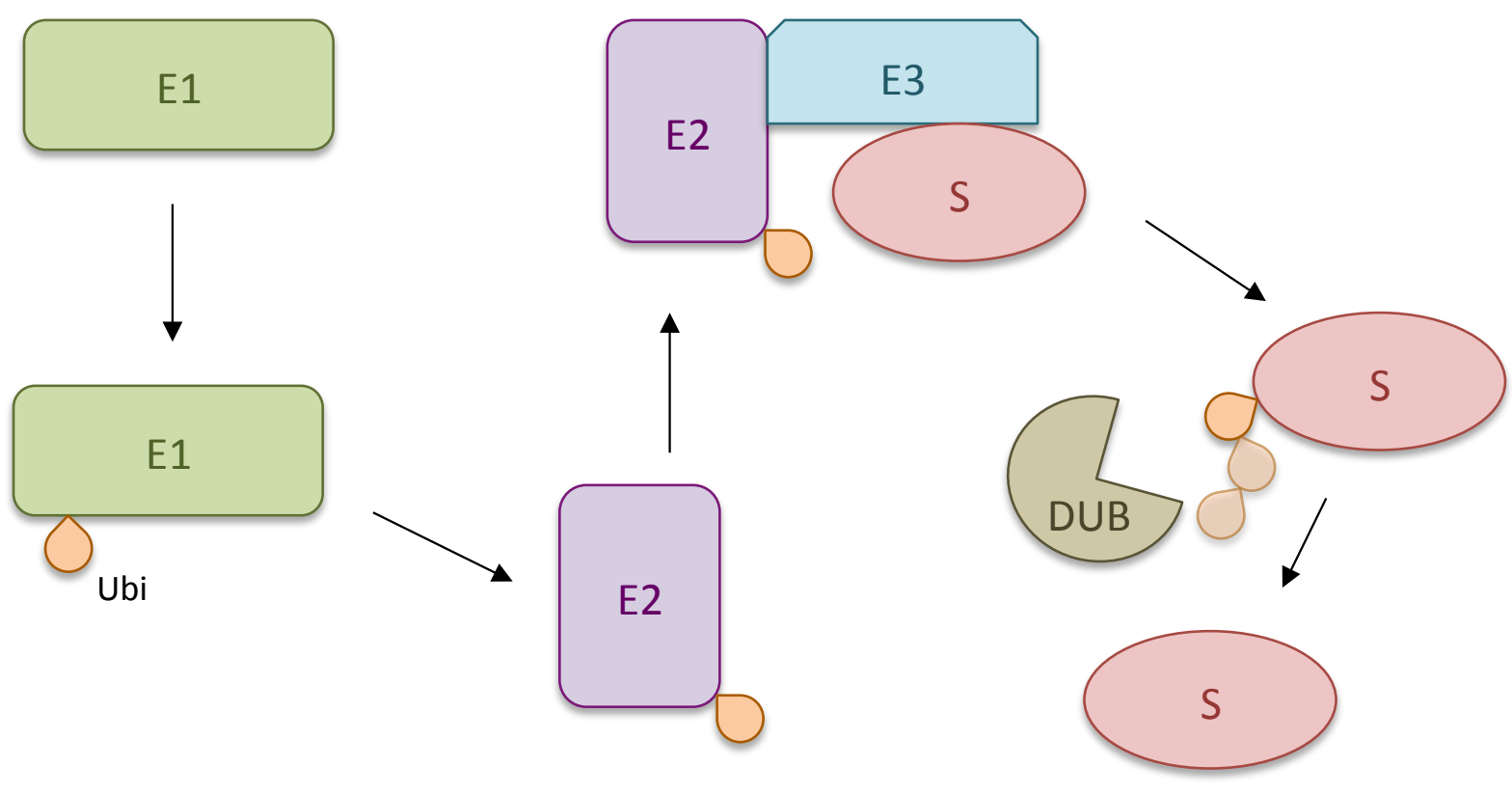

b

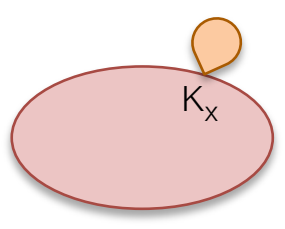

Monoubiquitylation

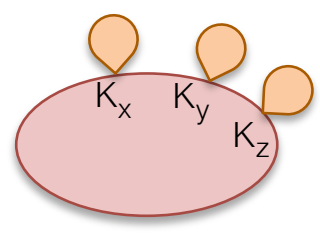

Multi-ubiquitylation

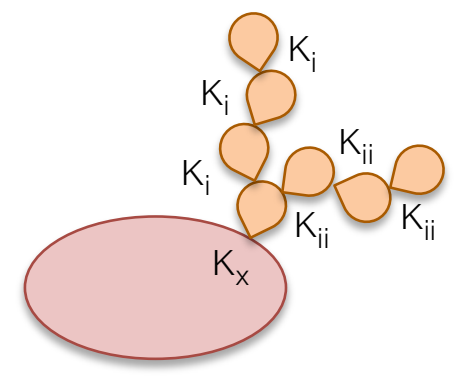

Branched polyubiquitylation

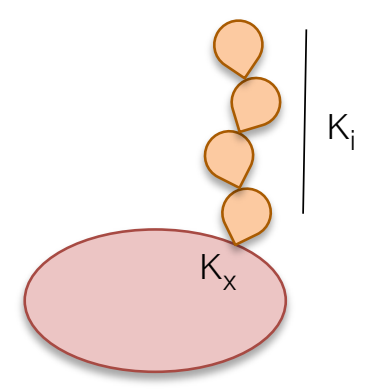

Polyubiquitylation

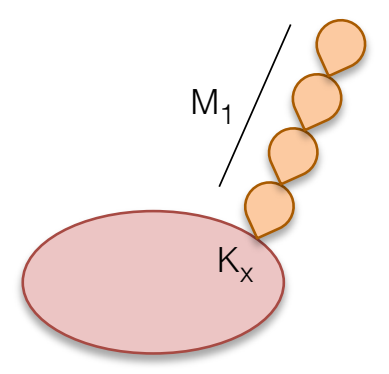

Linear polyubiquitylation

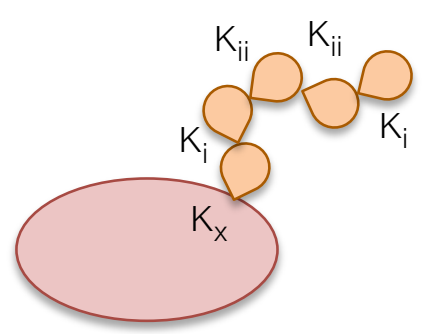

Mixed polyubiquitylation 
Figure 2

G1

ORC

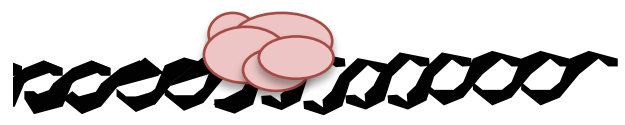
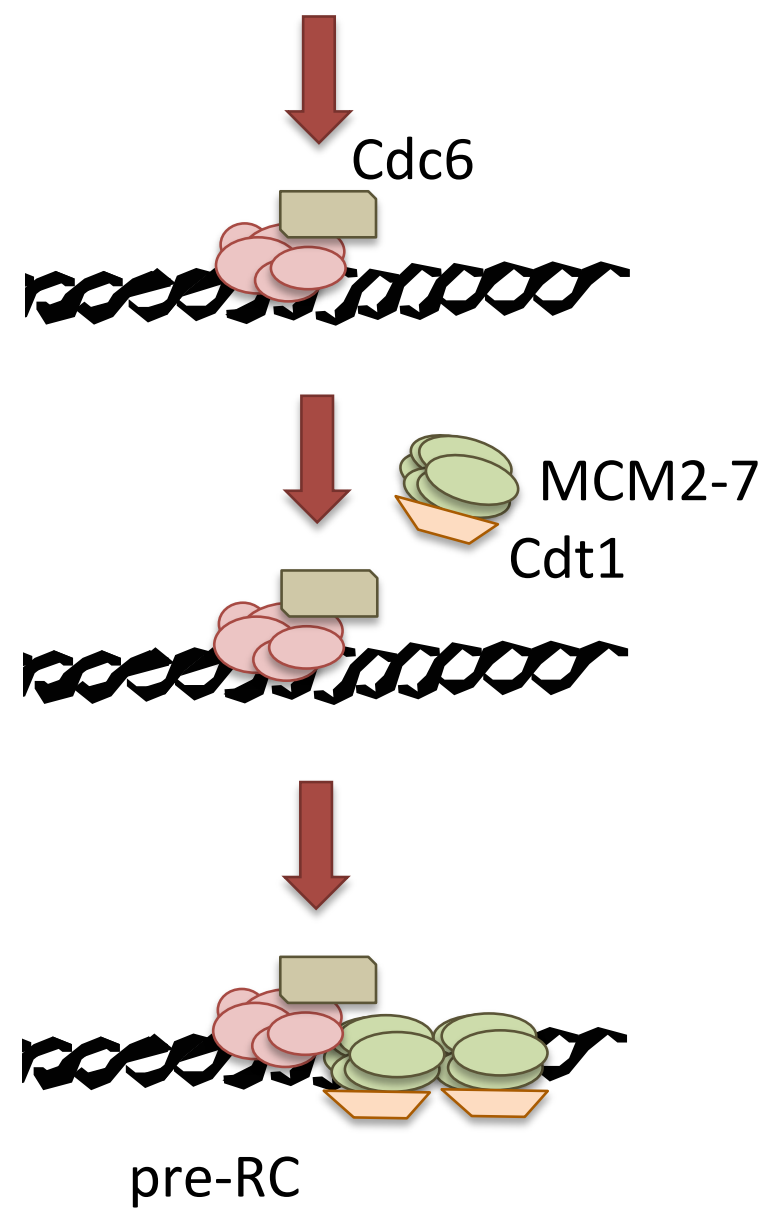

S-phase
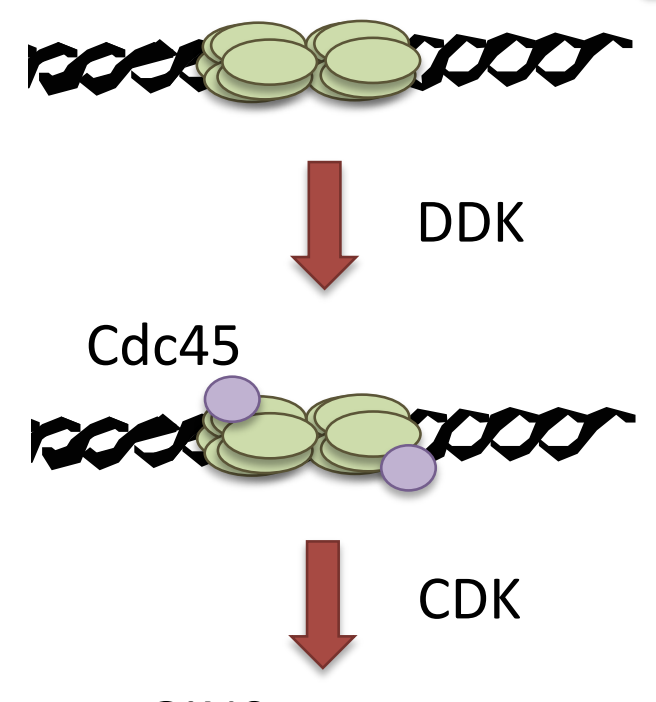

GINS

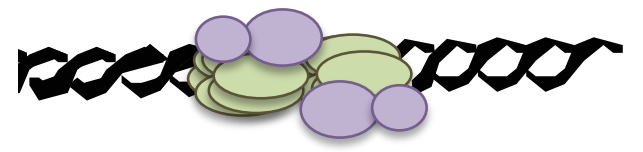

pre-IC
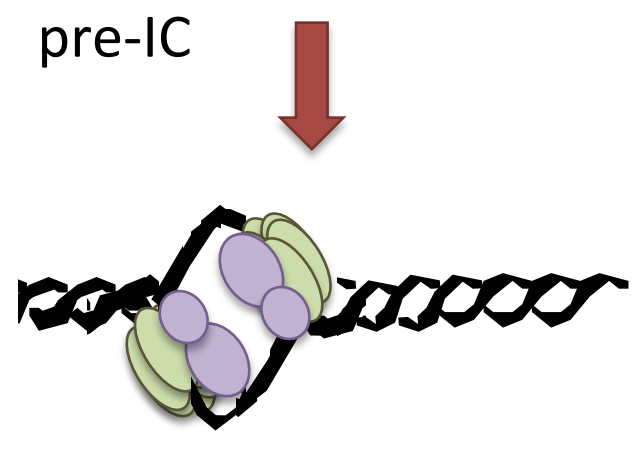
Figure 4

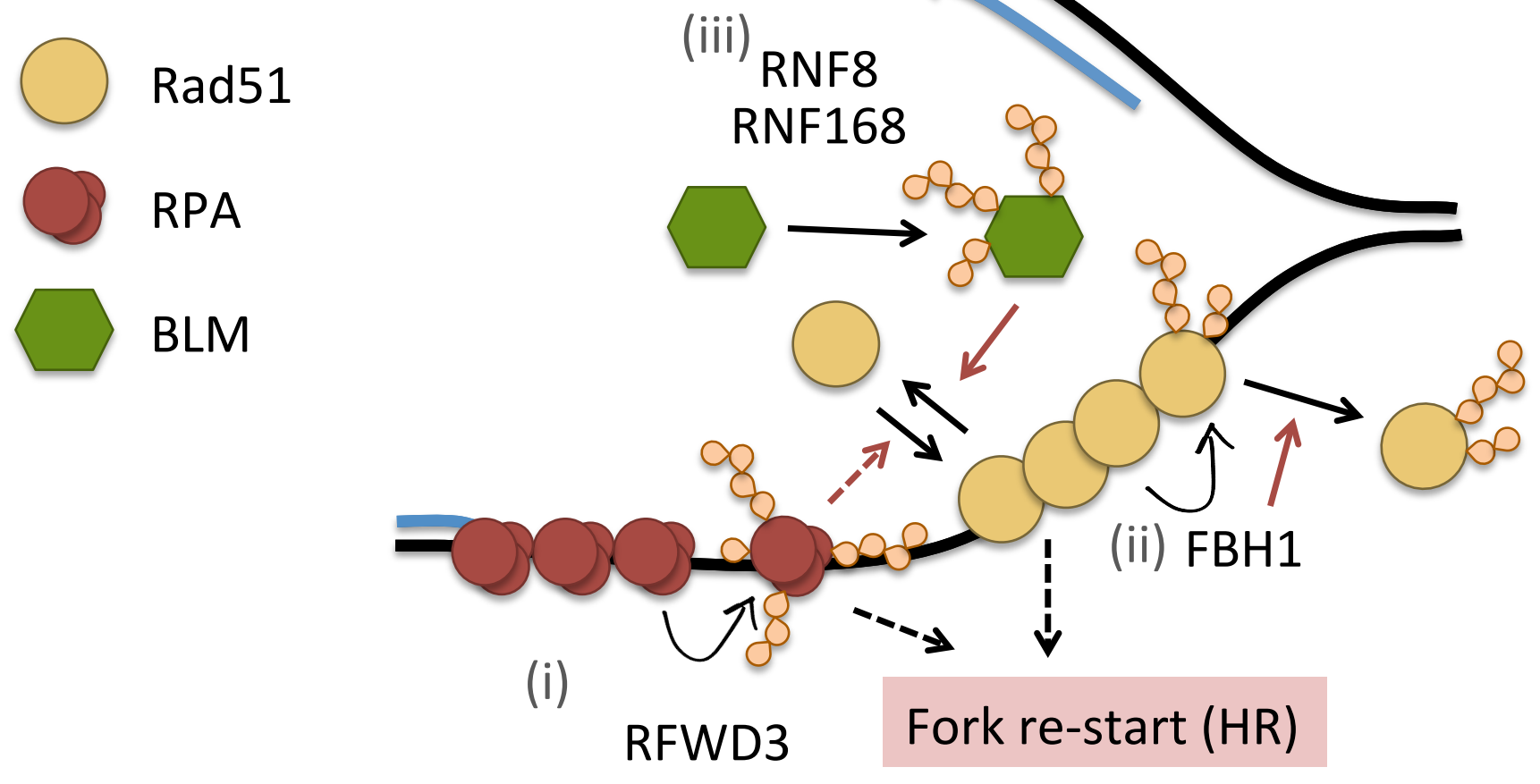


Figure 3

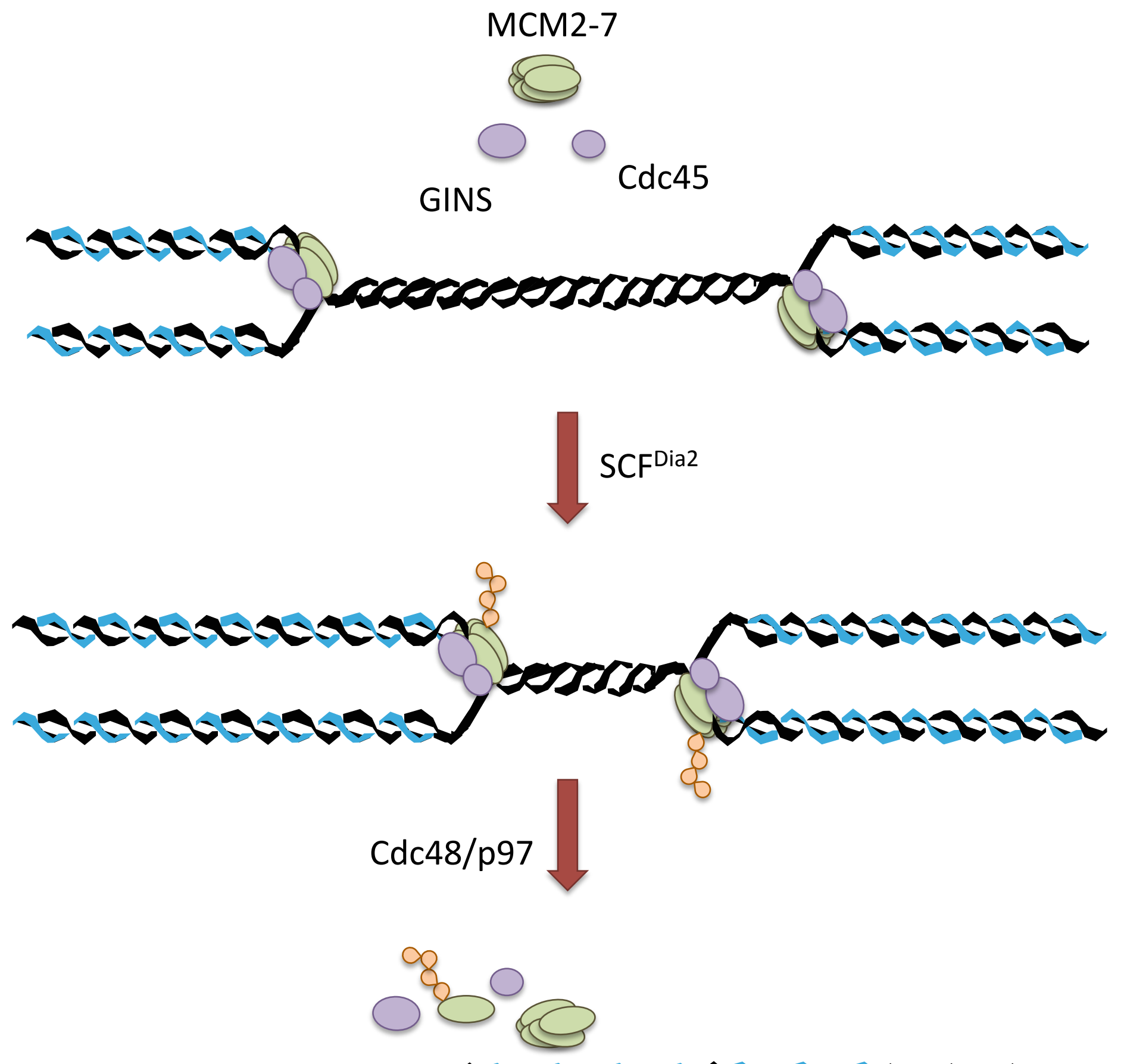

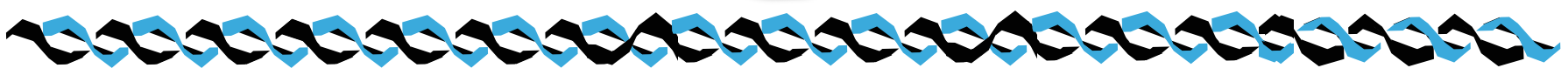

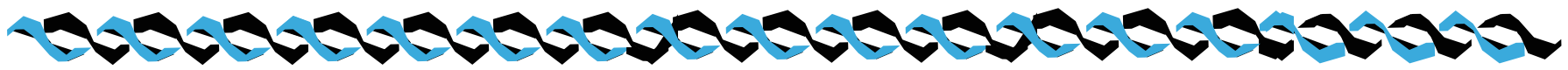

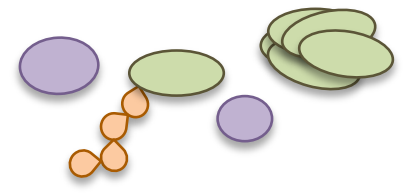


Figure 5

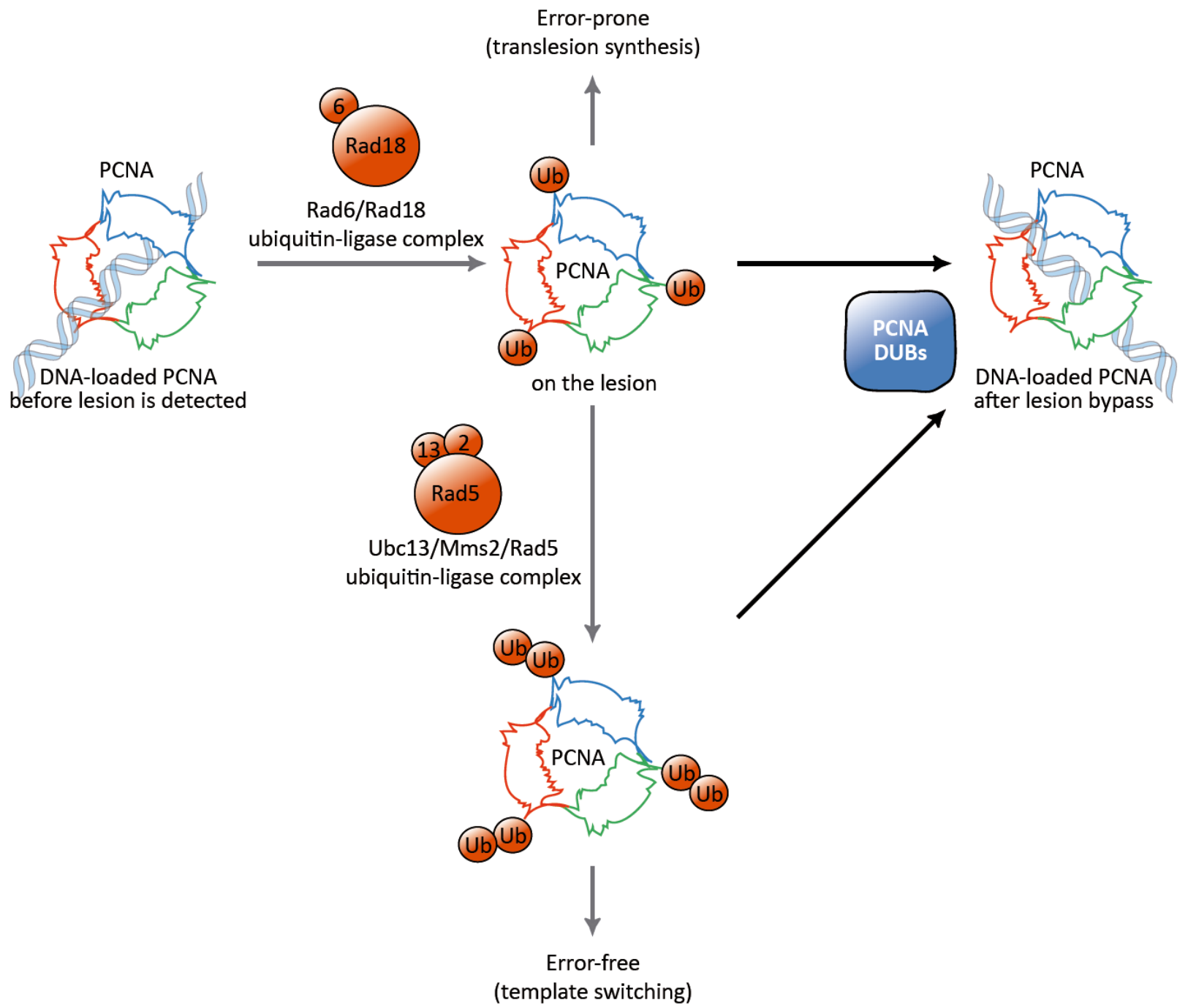


Figure 6
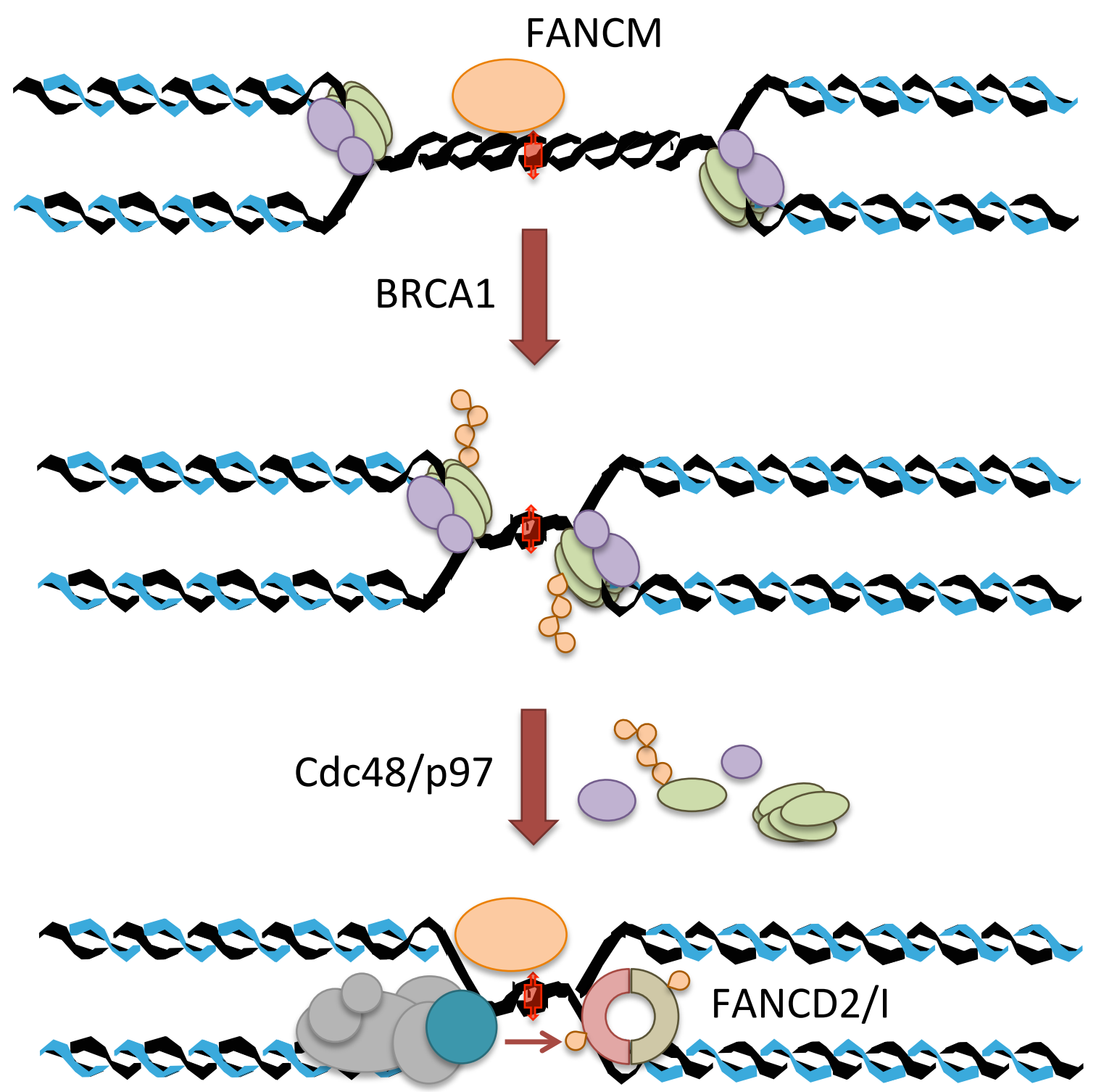

FANCL
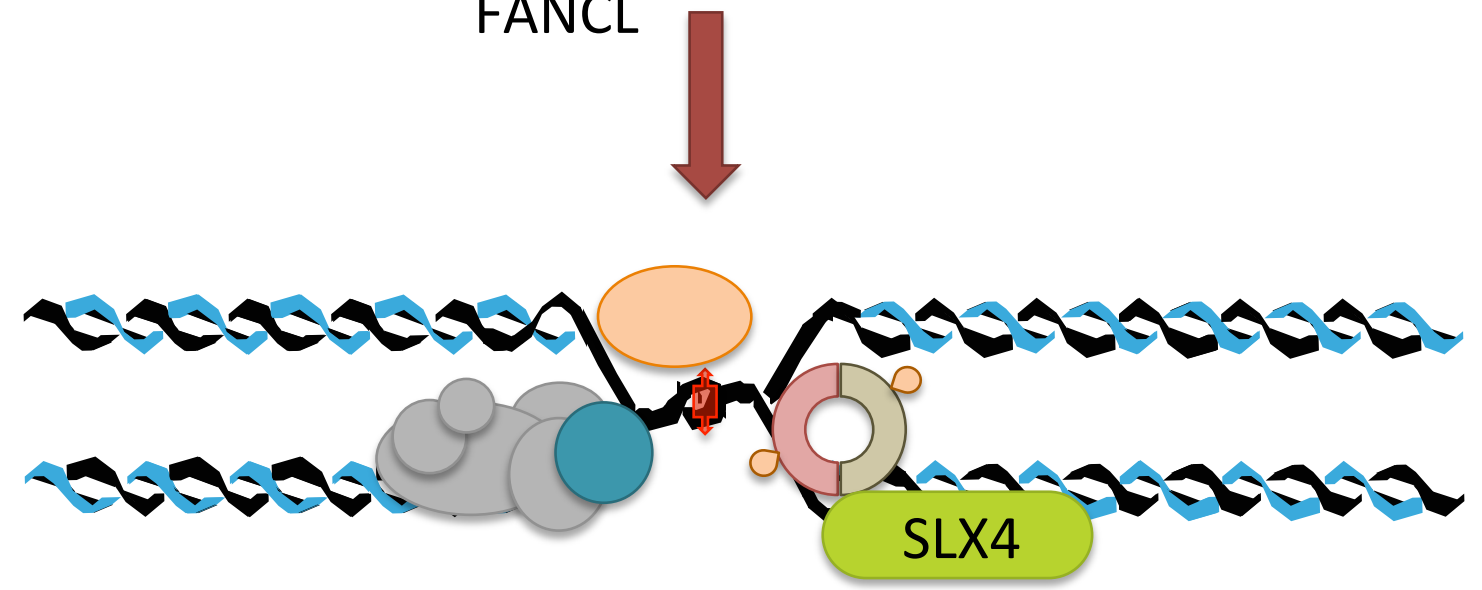

ICL incision - TLS - HR-repair 